\title{
Radiative Heating Methodology for the Huygens Probe
}

\author{
Christopher O. Johnston* \\ Virginia Tech, Blacksburg, VA, 24060 \\ Brian R. Hollis ${ }^{\dagger}$ \\ NASA Langley Research Center, Hampton, VA, 23681 \\ Kenneth Sutton \\ National Institute of Aerospace, Hampton, VA, 24060
}

\begin{abstract}
The radiative heating environment for the Huygens probe near peak heating conditions for Titan entry is investigated in this paper. The task of calculating the radiation-coupled flowfield, accounting for non-Boltzmann and non-optically thin radiation, is simplified to a rapid yet accurate calculation. This is achieved by using the viscous-shock layer (VSL) technique for the stagnation-line flowfield calculation and a modified smeared rotational band (SRB) model for the radiation calculation. These two methods provide a computationally efficient alternative to a Navier-Stokes flowfield and line-by-line radiation calculation. The results of the VSL technique are shown to provide an excellent comparison with the Navier-Stokes results of previous studies. It is shown that a conventional SRB approach is inadequate for the partially optically-thick

\footnotetext{
${ }^{*}$ NIA/NASA Graduate Research Assistant, Student Member AIAA

${ }^{\dagger}$ Aerospace Engineer, Senior Member AIAA 
conditions present in the Huygens shock-layer around the peak heating trajectory points. A simple modification is proposed to the SRB model that improves its accuracy in these partially optically-thick conditions. This modified approach, labeled herein as SRBC, is compared throughout this study with a detailed line-by-line (LBL) calculation and is shown to compare within 5\% in all cases. The SRBC method requires many orders-of-magnitude less computational time than the LBL method, which makes it ideal for coupling to the flowfield. The application of a collisional-radiative $(\mathrm{CR})$ model for determining the population of the $\mathrm{CN}$ electronic states, which govern the radiation for Huygens entry, is discussed and applied. The non-local absorption term in the CR model is formulated in terms of an escape factor, which is then curve-fit with temperature. Although the curve-fit is an approximation, it is shown to compare well with the exact escape factor calculation, which requires a computationally intensive iteration procedure.

\section{Nomenclature}

$$
\begin{array}{ll}
c & =\text { velocity of light, equal to } 2.997925 \times 10^{10} \mathrm{~cm} / \mathrm{s} \\
C_{r} & =\text { function of rotational temperature used in the smeared band model defined in Eq. (2) } \\
E_{J} & =\text { rotational term energy }\left(\mathrm{cm}^{-1}\right) \\
E_{V} & =\text { vibrational term energy }\left(\mathrm{cm}^{-1}\right) \\
E_{e} & =\text { electronic term energy }\left(\mathrm{cm}^{-1}\right) \\
e & =\text { electron charge, equal to } 4.80298 \times 10^{-10}\left(\mathrm{~cm}^{3 / 2} \cdot \mathrm{g}^{1 / 2} \cdot \mathrm{s}^{-1}\right) \\
F_{v} & =\text { incoming radiative intensity integrated over all directions }\left(\mathrm{erg} / \mathrm{cm}^{2}\right) \\
g_{x} & =\text { degeneracy of the energy mode } x
\end{array}
$$

\footnotetext{
$\$$ Senior Research Fellow, Associate Fellow AIAA
} 


$$
\begin{aligned}
& h \quad=\text { Planck's constant, equal to } 6.6256 \times 10^{-27} \mathrm{erg} \cdot \mathrm{s} \\
& I_{v} \quad=\text { frequency dependent radiative intensity }\left(\mathrm{erg} \cdot \mathrm{cm}^{-2} \cdot \mathrm{sr}^{-1}\right) \\
& j=\text { frequency integrated emission coefficient }\left(\mathrm{erg} \cdot \mathrm{cm}^{-3} \cdot \mathrm{s}^{-1} \cdot \mathrm{sr}^{-1}\right) \\
& j_{v}=\text { frequency dependent emission coefficient }\left(\mathrm{erg} \cdot \mathrm{cm}^{-3} \cdot \mathrm{sr}^{-1}\right) \\
& J \quad=\text { rotational quantum number } \\
& k=\text { Boltzmann constant, equal to } 1.38054 \times 10^{-16} \mathrm{erg} / \mathrm{K} \\
& k_{f, r}=\text { forward rate for collisional excitation defined in Eq. }(6)\left(\mathrm{cm}^{3} \cdot(\mathrm{g}-\mathrm{mol})^{-1} \cdot \mathrm{s}^{-1}\right) \\
& k_{b, r} \quad=\text { backward rate for collisional excitation defined in Eq. (7) }\left(\mathrm{cm}^{3} \cdot(\mathrm{g}-\mathrm{mol})^{-1} \cdot \mathrm{s}^{-1}\right) \\
& m \quad=\text { electron mass, equal to } 9.1091 \times 10^{-28} \mathrm{~g} \\
& N_{x} \quad=\text { number density of either a specie or a molecular state specified by the subscript } x \\
& \left(\text { particles } / \mathrm{cm}^{3}\right) \\
& N_{a} \quad=\text { Avogadro's number, equal to } 6.023 \times 10^{23} \text { particles } / \mathrm{g}-\mathrm{mole} \\
& Q_{x} \quad=\text { partition function of the energy mode } x \text { (nondimensional) } \\
& q_{r} \quad=\text { radiative heat flux, calculated in units of } \mathrm{erg} \cdot \mathrm{cm}^{-2} \cdot \mathrm{s}^{-1} \text {, but usually presented in units } \\
& \text { of } \mathrm{W} \cdot \mathrm{cm}^{-2} \text {, where } 1\left(\mathrm{erg} \cdot \mathrm{cm}^{-2} \cdot \mathrm{s}^{-1}\right)=10^{-7}\left(\mathrm{~W} \cdot \mathrm{cm}^{-2}\right) \\
& q_{c} \quad=\text { convective heat flux }\left(\mathrm{W} \cdot \mathrm{cm}^{-2}\right) \\
& S_{i m p, i}=\text { the rate of excitation of the level } i \text { due to impact with heavy particles and electrons } \\
& \left(\text { particles } \cdot \mathrm{cm}^{-3} \cdot \mathrm{s}^{-1}\right) \\
& S_{\text {rad,i }}=\text { rate of excitation of level } i \text { due to radiative emission and absorption } \\
& \left(\text { particles } \cdot \mathrm{cm}^{-3} \cdot \mathrm{s}^{-1}\right) \\
& T_{a} \quad=\text { dissociation temperature }(\mathrm{K}) \text {, equal to }\left(T_{t r} T_{v e}\right)^{1 / 2} \\
& T_{t r} \quad=\text { translational-rotational temperature }(\mathrm{K})
\end{aligned}
$$




$$
\begin{array}{ll}
T_{v e} & =\text { vibrational-electronic temperature }(\mathrm{K}) \\
V & =\text { vibrational quantum number } \\
z & =\text { distance along the stagnation line or through a constant-property slab }(\mathrm{cm}) \\
\Delta z & =\text { thickness of a constant-property slab }(\mathrm{cm}) \\
\Lambda_{l} & =\text { escape factor for electronic state } i \text { (nondimensional) } \\
\lambda & =\text { wavelength }(\mathrm{cm}) \\
v & =\text { frequency }\left(\mathrm{s}^{-1}\right) \\
v_{V} V^{\prime \prime} & =\text { frequency of a vibrational band head obtained from Eq }(\mathrm{B}-7) \text { of Ref. } 32\left(\mathrm{~s}^{-1}\right) \\
\tau_{l} & =\text { radiative lifetime for a transition with an upper state } i(\mathrm{~s}) \\
\text { Subscripts } & \\
e & =\text { refers to the electronic energy mode } \\
\text { inf } & =\text { refers to a free-stream value } \\
J & =\text { refers to the rotational energy mode } \\
V \quad & =\text { refers to the vibrational energy mode } \\
v & =\text { indicates frequency dependence }
\end{array}
$$

\section{Subscripts}

\section{Superscripts}

$$
\begin{aligned}
& -\quad=\text { indicates the radiative flux or intensity directed towards the vehicle wall } \\
& \text { “ } \quad=\text { defines a value in the upper electronic state of a transition } \\
& =\text { defines a value in the lower electronic state of a transition }
\end{aligned}
$$

\section{Introduction}

RECENT studies concerning the aerothermodynamic environment of the Huygens probe ${ }^{1,2}$, for 
entry into Titan, have indicated that significant uncertainty exists in the prediction of shocklayer radiative heating. A review of these studies leads to the conclusion that this uncertainty may be attributed to three primary sources: 1) the accuracy of the $\mathrm{CN}$ violet and red molecular band spectral representations (these band systems contribute the majority of the radiation at Huygens entry conditions); 2) the influence of radiation-flowfield coupling; 3) the accuracy of the kinetic scheme required for modeling the $\mathrm{CN}$ electronic state populations. The first of these was made clear by Hollis et al. ${ }^{1}$, who showed that two widely used radiation codes, NEQAIR and RAD/EQUIL, disagreed by a factor of two for Huygens conditions. Wright et al. ${ }^{2}$ have since shown agreement between the line-by-line approach of NEQAIR and other similar but independent line-by-line codes, which suggests that the lack of agreement shown by Hollis was due to inadequacies in the molecular band modeling of the RAD/EQUIL code. The uncertainty in the spectral modeling is therefore small if the line-by-line approach is used. The drawback to this approach is that it is very computationally expensive, which makes it very difficult to apply to a coupled radiation-flowfield analysis. Therefore, although it models the spectrum accurately for given flowfield conditions, the inaccuracy due to ignoring coupling remains (which was the second source of uncertainty listed above). The coupling effect has been approximately treated in these past studies using a correction factor ${ }^{3}$ developed for Jupiter entry. Unfortunately, this approximate method has not been validated for Titan entry with non-optically thin radiation. Further complications to the radiation calculation arise from the recent suggestion ${ }^{4}$ that the excited electronic states of the $\mathrm{CN}$ molecule, which are required for the radiation calculation, are not populated by a Boltzmann distribution. Removing the Boltzmann assumption requires a kinetic scheme, similar to that used for nonequilibrium chemistry modeling, to calculate the $\mathrm{CN}$ excited state populations. Kinetics schemes for $\mathrm{CN}$ excitation have been proposed recently by 
both Raynaud et al. ${ }^{5}$ and Magin et al. $^{6}$ specifically for Titan. It has been shown that implementing these models reduces the radiative heating significantly ${ }^{4-6}$. The influence of radiative absorption on the excitation calculation, as discussed by Bose et al. ${ }^{4}$, complicates the calculation further, especially if the spectrum is modeled using the line-by-line approach.

The aim of the present study is to formulate the Huygens probe radiative heating prediction, including all effects mentioned above, into a manageable calculation. A thermo-chemical nonequilibrium viscous-shock-layer analysis will be used for the flowfield calculation. The lineby-line method of calculating the radiative spectrum will be compared with less computationally intensive calculation methods at conditions of interest for the Huygens probe. A new approach to the spectrum calculation, which is a slight modification of the smeared-rotational band approach, will be discussed and validated. The radiative heating to the Huygens probe near peak heating conditions will be calculated using the developed flowfield and radiative models for both the coupled and uncoupled radiation cases. Finally, a method of implementing a collisionalradiative model to calculate the population of the $\mathrm{CN}$ electronic states will be presented and its effect on the radiative heating at Huygens peak heating conditions will be discussed.

\section{The Viscous Shock-Layer Stagnation Line Flowfield}

The flowfield was modeled in the present study using the viscous shock layer (VSL) method ${ }^{7}$. This method allows for a computationally efficient solution that retains all of the important flowfield physics for the stagnation region of a blunt body. Only the stagnation line flowfield was considered in the present study, although the same method may be used to obtain solutions downstream of the stagnation line. Chemical nonequilibrium was treated following the work of $\operatorname{Moss}^{8}$ and applying the reaction rates presented by Gocken ${ }^{9}$. Ionization effects have been shown to be negligible for Huygens entry, so the ions and electrons present in Gocken's model were not 
considered. This results in a model consisting of 14 species $\left(\mathrm{Ar}, \mathrm{C}, \mathrm{CH}, \mathrm{CH}_{2}, \mathrm{CH}_{3}, \mathrm{CH}_{4}, \mathrm{CN}, \mathrm{C}_{2}\right.$, $\mathrm{H}, \mathrm{HCN}, \mathrm{H}_{2}, \mathrm{~N}, \mathrm{NH}, \mathrm{N}_{2}$ ). Park's two temperature model ${ }^{10}$, derived for purely nitrogen freestream, was used to model vibrational nonequilibrium. This model assumes that the translational and rotational temperatures remain identical $\left(T_{t r}\right)$ and that the vibrational and electronic temperatures remain identical $\left(T_{v e}\right)$. Vibrational-dissociation coupling is treated in this model by defining a dissociation temperature, $T_{a}=\left(T_{t r} T_{v e}\right)^{1 / 2}$. The energy mode exchange and source terms required for the energy equations were taken from Gnoffo et al. ${ }^{11}$. This implementation of the two-temperature model into the VSL scheme followed the work of Gupta and Lee ${ }^{12}$ and Gupta ${ }^{13}$. Multicomponent diffusion was modeled using the "approximate-corrected" approach presented by Sutton and Gnoffo ${ }^{14}$. The Titan atmosphere was assumed to consist of $\mathrm{N}_{2}, \mathrm{CH}_{4}$, and $\mathrm{Ar}$ in mole fractions of $0.9699,0.0230$, and 0.0071 , respectively. An equivalent nose radius of $1.35 \mathrm{~m}$ was applied, which is slightly larger than probe's physical nose radius of $1.25 \mathrm{~m}$. This value was shown to give good agreement with the shock standoff and convective heating predicted by previous analyses ${ }^{1}$. A constant wall temperature of $2000 \mathrm{~K}$ was assumed for all cases and a wall emissivity of 0.9 was used for the radiation coupling calculation. This wall temperature was chosen to approximately match the radiative equilibrium wall values obtained in previous studies ${ }^{1}$. Therefore, the effect of radiation on the equilibrium wall temperature, which should have a negligible influence on the flowfield calculation, is not accounted for in this study. The wall was also assumed to be super-catalytic, meaning the shock-layer species at the wall were set equal to their free-stream values.

The present study will concentrate on the three peak heating trajectory points identified in previous studies ${ }^{1}$. These conditions are shown in Table 1. Other trajectory points have been 
considered throughout the course of this work, but to allow a focused and thorough discussion of the most severe conditions, this paper will focus exclusively on these three peak heating points.

The flowfield solutions obtained with the VSL method developed for this study were found to compare well with the Navier-Stokes solutions of previous studies ${ }^{1}$. Figure 1 presents the temperature profiles for the three trajectory points. The discrete-shock used in the VSL method results in the translational-rotational temperature $\left(T_{t r}\right)$ directly behind the shock being larger than what would be found using a shock-capturing method. This temperature overshoot has a negligible effect on both the radiative and convective heating. Figure 2 presents the $\mathrm{CN}$ number densities for the three trajectory points.

\section{Spectral Modeling of CN Molecular Band Radiation}

Various methods are available for the general modeling of molecular band spectra. The method chosen for determining the radiative flux from a hypersonic shock-layer is required primarily to model the frequency-integrated flux to the vehicle wall. Spectral details that do not contribute noticeably to the integrated flux are not of interest, and should be ignored if they require significant computational effort. The two methods that will be considered here are the line-by-line (LBL) approach, as presented by Arnold and Whiting ${ }^{15}$ and used in NEQAIR ${ }^{16,17}$, and the smeared-rotational band (SRB) approach, as derived by Patch, Shackleford, and Penner ${ }^{18}$ and extended to higher order accuracy and multiple temperatures by Chambers ${ }^{19,20}$. The details of the LBL approach for $\mathrm{a}^{2} \Sigma-{ }^{2} \Sigma$ transition, which is consistent with the $\mathrm{CN}$ violet $\left(\mathrm{X}^{2} \Sigma^{+}-\mathrm{B}^{2} \Sigma^{+}\right)$ transition, are presented in Appendix A of Ref. 32. The details of the SRB approach are presented in Appendix B of Ref. 32. The spectroscopic constants and absorption oscillator strengths $\left(f_{V^{\prime} V^{\prime \prime}}\right)$ for the $\mathrm{CN}$ violet and red transitions were taken from Laux ${ }^{21}$ for the present study. 
The emission coefficients for the $\mathrm{CN}$ red band, and the resulting radiative flux, presented in this paper are $1 / 2$ of the values presented in an earlier version of this paper $^{32}$. This is a result of the missing $g_{e^{\prime \prime}} / g_{e^{\prime}}$ factor in Eq. (B-2) of Ref. 32 (fortunately, this factor is equal to one for $\mathrm{CN}$ violet). The corrected values for the $\mathrm{CN}$ red radiation have been included throughout the present paper, including the radiation-coupled cases, although because of the minor importance of the $\mathrm{CN}$ red radiation for Huygens entry, this did not change any of the conclusions reached previously in Ref. 32 .

Assuming that it is implemented correctly and that accurate spectroscopic data is used, the LBL approach is considered to provide the theoretically exact radiative spectrum for a molecule. As a result, it provides an accurate value for the spectrally-integrated radiative flux, regardless of the optical depth. The drawback to this method is that it requires an enormous number of spectral points (on the order of 100,000 for the spectral range of the $\mathrm{CN}$ violet band). This makes it very difficult to apply in a coupled radiation-flowfield calculation, where many radiation calculations are required. The SRB method, on the other hand, produces an approximate spectrum that captures the average shape of each vibrational band $\left(V^{\prime}-V^{\prime \prime}\right)$. This provides a rough estimate of the spectrum while maintaining an accurate spectrally-integrated radiative flux in the optically thin limit. The accuracy of the SRB method in the optically-thin limit is a result of the method's derivation, which forces this to be true for any molecular band system. Figure 3 compares the LBL and SRB values for the integrated emission coefficient, which is proportional to the integrated flux in the optically thin limit, for the $\mathrm{CN}$ violet band over a range of temperatures. The contribution from each of the major three vibrational bands is shown separately along with the total. In all cases, good agreement is seen between the SRB and the LBL values. This 
confirms the statement that the SRB method is a good approximation of the LBL method for an optically thin gas.

To examine how the methods compare as the gas becomes more optically thick, Table 2 compares the integrated intensity from a $1-\mathrm{cm}$ slab of $\mathrm{CN}$ in equilibrium at $7000 \mathrm{~K}$ in spectral range of 3.176 to $3.444 \mathrm{eV}$ for various pressures. The entire $\Delta V=0$ band and a small part of the $\Delta V=1$ band are captured in this spectral range. This comparison was also made by Wright et $\mathrm{al}^{2}$, whose values are shown in the "NEQAIR96" column of the table. The NEQAIR96 values, which are from a line-by-line calculation, compare well with the current LBL results. A comparison was also made between the present LBL results and the NEQAIR96 results for the intensity resulting from spectral intervals of 25 Angstrom. The two values were shown to compare within $5 \%$ for each interval. This excellent comparison provides confidence that the present LBL calculation has been implemented correctly. As for the comparison between the LBL and SRB values, Table 2 shows that they do not compare well for the two higher pressure cases. This suggests that the SRB method is inadequate for modeling a gas with optically thick spectral regions. The optical thickness of a constant property slab is dependent on the term $N_{C N} \Delta z$, where $N_{C N}$ is the $\mathrm{CN}$ number density and $\Delta z$ is the thickness of the slab. This term is a similarity parameter for the radiative flux emitted from a constant property slab of $\mathrm{CN}$ in equilibrium. Taking the shock-layer thickness to be about 10-cm from Figure 1 and $N_{C N}$ to be about $1 \times 10^{15} \mathrm{~cm}^{-3}$ from Figure 2, a constant property slab representative of Huygens peak heating conditions is found to have a $N_{C N} \Delta z$ equal to about $1 \times 10^{16} \mathrm{~cm}^{-3}$. Figure 4 compares the LBL and SRB result in a small region of the spectrum (3.19-3.21 eV) for the 0.001 and 0.01 atmosphere cases, which correspond to values of $N_{C N} \Delta z$ equal to $1 \times 10^{15}$ and $1 \times 10^{16} \mathrm{~cm}^{-2}$, respectively. It is seen in these comparisons that the SRB model does not capture the intense 
peaks predicted by the LBL model at each vibrational band head. This has a small influence on the integrated flux for the $0.001 \mathrm{~atm}$ case $\left(N_{C N} \Delta z=1 \times 10^{15} \mathrm{~cm}^{-2}\right)$ because even the largest peaks of the LBL spectrum are less than $75 \%$ of the blackbody limit (which is represented by $\pi B_{v}$ in the figure) so that the entire spectrum is essentially optically thin. For the $0.01 \mathrm{~atm}$ case $\left(N_{C N} \Delta z\right.$ $\left.=1 \times 10^{16} \mathrm{~cm}^{-2}\right)$, it is seen that the vibrational band heads predicted by the LBL method are suppressed by the blackbody limit, and much of the nearby spectrum is near this limit. These spectral regions are therefore optically thick. The SRB prediction for this case, on the other hand, fails to predict any spectral region that is more than half of the blackbody limit. The predicted spectrum therefore remains optically thin, which means that the absorption and corresponding reduction in flux predicted by the optically thick LBL method is not properly modeled by the SRB method. As a result, the spectrally integrated flux listed in Table 2 for the SRB model is larger than the LBL prediction, and the comparison between the two methods gets worse as the optical thickness increases.

The previous discussion indicated that the SRB method does not provide a satisfactory model for the spectrum at Huygens peak heating conditions. Through the course of the present work, a simple correction to this model was developed that considerably improves its modeling capability. To rationalize the correction developed for the SRB method, it is first observed from the equations presented in Appendix B of Ref. 32 that decreasing the rotational temperature $\left(T_{r}\right)$ increases the magnitude of each vibrational band head. This implies that the spectrum has larger spikes, which is the behavior required for a better agreement with the LBL results. Since this method is an approximation to begin with, it is justifiable to consider the possibility of choosing a nonphysical value of $T_{r}$ to improve the method's agreement with the LBL results. This is equivalent to introducing an empirically derived correction factor into the SRB equations 
wherever $T_{r}$ appears, and does not imply that the physical value of the rotational temperature is believed to be any different than what is predicted by the flowfield solution (where it is assumed equal to the translational temperature). The initial concern in attempting this approach is that it may reduce the accuracy of the SRB method in the regime in which it is already known to be accurate, which was shown previously in Figure 3 to be the optically thin limit. Therefore, for this approach to be valid, it must be shown that the rotational temperature has a small influence on the frequency-integrated emission coefficient $(j)$. The integrated emission coefficient from a single vibrational band $\left(j_{V^{\prime} V^{\prime}}\right)$ may be evaluated analytically for the SRB method as follows

$$
\begin{aligned}
j_{V^{\prime} V^{\prime \prime}} & =\frac{N_{e^{\prime}}}{Q_{V^{\prime}} Q_{J^{\prime}}} \exp \left(-\frac{h c}{k T_{v}} E_{V^{\prime}}\right) \frac{2 \pi e^{2} h}{c^{4} m} \frac{g_{e^{\prime \prime}}}{g_{e^{\prime}}} \frac{f_{V^{\prime} V^{\prime \prime}}}{\mid B_{e^{\prime}}-B_{e^{\prime \prime}}} \int_{V_{V^{\prime \prime}}}^{\infty} v^{3} \exp \left(-C_{r}\left(v-v_{V^{\prime} V^{\prime \prime}}\right)\right) d v \quad\left(\frac{e r g}{c m^{3} \cdot s \cdot s r}\right) \\
& =\frac{N_{e^{\prime}}}{Q_{V^{\prime}}} \exp \left(-\frac{h c}{k T_{v}} E_{V^{\prime}}\right) \frac{2 \pi e^{2} h}{c^{3} m} \frac{g_{e^{\prime \prime}}}{g_{e^{\prime}}} f_{V^{\prime} V^{\prime \prime}}\left[\nu_{V^{\prime} V^{\prime \prime}}{ }^{3}-\frac{3}{C_{r}} v_{V^{\prime} V^{\prime \prime}}{ }^{2}-\frac{6}{C_{r}{ }^{3}}\left(C_{r} v_{V^{\prime} V^{\prime \prime}}-1\right)\right]
\end{aligned}
$$

where

$$
C_{r}=\frac{B_{V^{\prime}}}{B_{V^{\prime}}-B_{V^{n}}} \frac{h}{k T_{r}}
$$

and $v_{V^{\prime} V^{\prime}}$ is the frequency of the vibrational band head. The other parameters in this equation are defined in the Nomenclature section. The useful result of this equation is that the underlined terms are the only place where the rotational temperature appears. This is significant because the underlined terms are negligible relative to the $v_{V}, V^{,},{ }^{3}$ term for any practical case. This is because $v_{V^{\prime} V^{\prime}}$, is usually much larger that $1 / C_{r}\left(\nu_{V^{\prime} V^{\prime \prime}} \sim 25,000 \mathrm{sec}^{-1}\right.$ for the $\mathrm{CN}$ violet band while $1 / C_{r} \sim$ $\left.170 \mathrm{sec}^{-1}\right)$. It is therefore shown that the rotational temperature has a very small influence on the integrated emission, which was the desired result. The next step is to find the value for $T_{r}$ which provides the best fit to the LBL calculation over a wide range of conditions. By investigating numerous constant property slabs over a range of temperatures and optical depths, the best value 
was found to be $T_{r}=1700 \mathrm{~K}$. The results obtained using this approach will be referred to throughout this paper as the smeared rotational band corrected model, or SRBC. An example of the spectrum produced by this approach is shown in Figure 4. As intended, the vibrational band head peaks are much larger and more closely imitate the LBL spectrum. Figure 5 compares the flux from a constant-property slab at various optical depths and two different temperatures. The results consider the radiation from only the $\mathrm{CN}$ violet $\Delta V=0$ region of the spectrum, which is the most optically thick. The success of the SRBC method is clearly seen throughout the entire range of conditions. This result is characteristic of the other conditions investigated, which included temperatures ranging from 3000 to $9000 \mathrm{~K}$. As mentioned previously, the constant property slab characteristic of the shock layer at Huygens peak heating conditions requires a value of $N_{C N} \Delta z$ no larger than $1 \times 10^{16} \mathrm{~cm}^{-2}$. Therefore, the SRBC model is more than adequate for the present application.

\section{Uncoupled and Coupled Radiative Heating Assuming a Boltzmann Distribution}

The SRBC model was shown in the previous section to accurately predict the radiative flux for conditions of present interest. The use of the SRBC approach, rather than the LBL approach, results in a significant decrease in the computational burden associated with radiation coupling. But, to be completely sure that the SRBC model is providing accurate results, the SRBC and LBL approach will be used here for the uncoupled calculations. This allows the accuracy of the SRBC method to be monitored relative to the LBL calculations for an actual shock layer, and not just a constant property slab. For the coupled calculations, only the SRBC approach is used. However, once a converged coupled flowfield is obtained, the LBL approach is used to check the validity of the SRBC in the new flowfield. Note that no correction has been included in the 
presented results to account for shock-layer curvature effects. In Ref. 2 a correction is applied by multiplying the radiative heat flux by 0.8 .

Table 3 presents a breakdown of the uncoupled radiation spectrum for the three trajectory points considered. The values in parentheses are the LBL results, while the values not in parentheses are the SRBC results. This convention will be used throughout the rest of this paper. Note that the $\mathrm{CN}$ red spectrum was only calculated using the SRBC approach. This table shows good agreement between the two methods for both the total flux value and the individual components. The distribution through the shock layer of each of these components, predicted by the SRBC approach for the $t=189$ case, is shown in Figure 6. The corresponding radiative flux spectrum at the wall for the $\mathrm{CN}$ violet band is shown in Figure 7. It is seen that the different $\Delta V$ bands do not overlap, which means that they may be treated independently in the spectrum and transport calculation. This is also true for the $\mathrm{CN}$ red band as a whole, which is located around 1 $\mathrm{eV}$. The radiative heating values predicted by previous studies ${ }^{1}$ are also listed in Table 3 for comparison. The RAD/EQUIL result is much larger than the current prediction is because it models the molecular band spectrum with a curve-fit over frequency, which is even more of an approximation than the SRB model. The blackbody limiting effect is therefore under predicted because the spectrum is too smooth, similarly to the SRB model. The agreement of the current predictions with the NEQAIR results is excellent, especially considering that different flowfield models were applied. The smaller contribution from the $\mathrm{CN}$ red band presented here, relative to those presented in the earlier version of this paper ${ }^{32}$, improved the agreement with the NEQAIR results considerably. Note that the study by Olejniczak et al. ${ }^{22}$, using NEQAIR, ignored the CN red contribution entirely. 
As mentioned previously, radiation-coupled flowfields were obtained using the SRBC approach for the radiation model. This computationally efficient approach allowed coupled flowfields to be obtained without making any assumptions regarding the optical thickness, as was done in previous studies ${ }^{23,24}$. A discussion and comparison of the coupled flowfields will be presented here, followed by a discussion of the coupling effect on the radiative heating. Figure 8 compare the temperature profiles for the $t=189$ uncoupled and coupled cases. The expected trend for the coupled case of reduced temperature and shock standoff distance is seen. Figure 9 compares the $\mathrm{CN}$ number density profiles for the coupled and uncoupled cases. The interesting result of increased $\mathrm{CN}$ number density with the addition of coupling is found. The explanation of this phenomenon requires the study of the contribution of each chemical reaction that involves $\mathrm{CN}$ to the total mass rate of formation of $\mathrm{CN}$. Figure 10 plots the mass rate of formation of $\mathrm{CN}$ resulting from three of the main reactions involving CN. Although Gocken's ${ }^{9}$ simplified reaction set contains 6 reactions involving $\mathrm{CN}$, the three not presented here do not contribute significantly to the difference between the coupled and uncoupled cases, although their contribution is included in the "Total" line presented in Figure 10. The reason for the increased CN number density for the coupled case is seen from this figure to be a result of the smaller negative formation values from reactions 1 and 3. Because these two reactions involve $\mathrm{CN}$ as a reactant, the lower temperatures throughout most of the shock layer for the coupled case results in a decreased forward reaction rate, and therefore lower rate of $\mathrm{CN}$ destruction. Reaction 2 has the opposite effect, but its influence is overshadowed by reactions 1 and 2 . The large positive formation values near the shock reach almost exactly the same peak for the coupled and uncoupled case, which explains the similar number density profiles seen near the shock in Figure 9. 
Table 4 compares the convective heat flux at the wall for the coupled and uncoupled cases. A reduction of 11 to $15 \%$ is found for the three trajectory points. The influence of radiation on the convective heating is a combination of two competing mechanisms. The absorption of radiation in the boundary layer tends to increase the convective heating, while the emission of radiation out of the shock layer away from the body tends to decrease it. The mild decease in convective heating predicted here is consistent with the previous observation that the shock layer is optically thick only in the small spectral region of the $\mathrm{CN}$ violet vibrational band heads. Note that the values for the uncoupled case agree within $5 \%$ of the Navier-Stokes results presented by Hollis ${ }^{1}$.

Table 5 presents the wall radiative heat flux values for the coupled case and compares them with the uncoupled values presented previously. Note that the values in parentheses are the LBL results that were calculated only after the coupled flowfield solution was obtained using the SRBC approach. An observed reduction in the flux values ranging from 18 to 25 percent indicates the significant influence of coupling on the radiation calculation. This reduction also indicates that although the $\mathrm{CN}$ number density was shown to increase with the addition of coupling, its effect on the radiation is overshadowed by the corresponding temperature decrease. This is because the emission coefficient depends exponentially on temperature, assuming a Boltzmann distribution, and only linearly on the $\mathrm{CN}$ number density. The column labeled "coupled-TW" presents the coupled radiative heating predicted using the Tauber-Wakefield approximate method ${ }^{3}$. This method depends on the uncoupled radiative heating and a constant, $\kappa$, which has been assumed equal to 2 in previous studies ${ }^{1,2}$. The present results show that this method over predicts the effect of coupling for the first two trajectory points. Figure 11 shows the difference in the wall directed radiative flux profiles with the addition of coupling. Although the magnitudes of the coupled results are lower than the uncoupled results, the shapes of the 
profiles are similar. This indicates that the temperature decrease due to the addition of coupling does not introduce any fundamental changes in the radiation spectrum and transport, other than the expected decrease in emission.

\section{Collisional-Radiative Modeling for Titan Entry}

Recent shock-tube experiments by Bose et al. ${ }^{4}$ have shown that near Huygens peak heating conditions, the measured $\mathrm{CN}(\mathrm{B})$ population $^{\S}$ is an order-of-magnitude less than predicted by a Boltzmann distribution. Since the $\mathrm{CN}(\mathrm{B})$ state is the upper level of the $\mathrm{CN}$ violet transition, this implies that the radiation from the $\mathrm{CN}$ violet band is an order-of-magnitude less than that predicted assuming a Boltzmann distribution. Using excitation rates from previous studies ${ }^{25,26}$, Bose et al. ${ }^{4}$ formulated a simple collisional-radiative (CR) model that provided a closer prediction to their experimental results than the Boltzmann model. Raynaud et al. ${ }^{5}$ and Magin et al. ${ }^{6}$ have since assembled a more elaborate set of excitation rates and discussed their influence on the predicted $\mathrm{CN}(\mathrm{B})$ population. The present study will review the rates suggested by these past studies and consider the effect of using other values found in the literature. Also, the absorption term shown by Bose to be important, will be formulated in terms of an escape factor. It will be shown that this term can then be curve fit as a function of temperature and therefore implemented easily into the $\mathrm{CR}$ model. This avoids the costly iteration procedure used by Bose et al. in their treatment of the absorption term. The details of the CR model as implemented in the present study are discussed below.

The conservation equation for the electronic state $i$ of a molecule may be written as

$$
\frac{\partial N_{i}}{\partial t}=S_{i m p, i}+S_{r a d, i} \quad\left(\frac{\text { particles }}{\mathrm{cm}^{3} \cdot s}\right)
$$

\footnotetext{
${ }^{\S}$ The notation that $\mathrm{CN}(\mathrm{B})$ refers to $\mathrm{CN}\left(\mathrm{B}^{2} \Sigma^{+}\right), \mathrm{CN}(\mathrm{X})$ refers to $\mathrm{CN}\left(\mathrm{X}^{2} \Sigma^{+}\right)$, and $\mathrm{CN}(\mathrm{A})$ refers to $\mathrm{CN}\left(\mathrm{A}^{2} \Pi\right)$ will be used throughout this paper.
} 
where the term on the left is the time derivative of the number density (particles $/ \mathrm{cm}^{3}-\mathrm{s}$ ) of the molecules in the electronic state $i, S_{\text {imp, } i}$ is the rate of excitation of the level $i$ due to impact with heavy particles and electrons, and $S_{r a d, i}$ is the rate of excitation of level $i$ due to radiative emission and absorption. The $S_{i m p, i}$ term is written as follows

$$
S_{\text {imp }, i}=\frac{1}{N_{a}} \sum_{r=1}^{N_{r}}\left(\beta_{i, r}-\alpha_{i, r}\right)\left(k_{f, r} N_{A(1)} N_{B(1)}-k_{b, r} N_{A(2)} N_{B(2)}\right) \quad\left(\frac{\text { particles }}{\mathrm{cm}^{3} \cdot s}\right)
$$

where $\alpha_{i, r}$ and $\beta_{i, r}$ are the forward and backward stoichiometric coefficients for reaction $r$, respectively. This equation assumes that the excitation processes may be written in the following form

$$
\mathrm{A}(1)+\mathrm{B}(1) \longleftrightarrow \mathrm{A}(2)+\mathrm{B}(2)
$$

where $\mathrm{A}$ and $\mathrm{B}$ each represent a specie, which may be the same or different, and 1 and 2 represent the initial and final electronic state of the specie, respectively. In Eq. (4), the $N$ values with either an A or B subscript represent the number density of the particular state. Table 6 lists the excitation processes, and corresponding $\operatorname{rates}^{5,6}$, which were found to contribute noticeably to the $\mathrm{CN}(\mathrm{B})$ population. The electron impact excitation rates are not required for the weaklyionized cases considered here, but they are listed because they may be important for other cases. Note that Magin et al. ${ }^{6}$ found these reactions to have a significant effect at the present flow conditions because their flowfield contained a significantly higher electron number density than what is currently predicted (when the ionized species are considered in the flowfield). This over prediction of electrons was a result of their use of Nelson's ${ }^{27}$ chemical reaction rate set instead of Gocken's ${ }^{9}$ recent model. The influence of other possible values for the rates listed in Table 6 will be discussed later. The forward rate coefficient is defined in terms of the listed parameters as 


$$
k_{f, r}=A_{r} T_{f}^{n_{r}} \exp \left(-C_{r} / T_{f}\right) \quad\left(\frac{\mathrm{cm}^{3}}{g-m o l e \cdot s}\right)
$$

Considering reactions of the form of Eq. (4), the backwards rate coefficient may be written from detailed balancing as

$$
k_{b, r}=k_{\mathrm{f}, \mathrm{r}}\left(T_{b}\right) \frac{g_{\mathrm{A}(1)} g_{\mathrm{B}(1)}}{g_{\mathrm{A}(2)} g_{\mathrm{B}(2)}} \exp \left[\frac{h c}{k T_{b}}\left(E_{A(2)}-E_{A(1)}+E_{B(2)}-E_{B(1)}\right)\right]
$$

where $g$ and $E$ represent the degeneracy and term energy $(1 / \mathrm{cm})$ of the indicated state, respectively.

The rate of excitation of level $i$ due to radiative emission and absorption, $S_{\text {rad,i }}$, may be written as

$$
S_{\text {rad }, i}=-\frac{N_{i}}{\tau_{i}} \Lambda_{i} \quad\left(\frac{\text { particles }}{\mathrm{cm}^{3} \cdot s}\right)
$$

where $\tau_{i}$ is the radiative lifetime in seconds, which are listed in Table 7 for $\mathrm{CN}$, and $\Lambda_{i}$ is the escape factor (nondimensional). As mentioned previously, the escape factor accounts for the radiative absorption term shown by Bose et al. ${ }^{4}$ to be important for Huygens conditions. If absorption is ignored, or if the gas is optically thin, the escape factor is equal to one. This escape factor is shown in Appendix C of Ref. 32 to be written as

$$
\Lambda_{i}=1-\frac{\tau_{i}}{N_{i}} \int_{0}^{\infty} \frac{\kappa_{v, i}^{*}}{h v} F_{v} d v
$$

where $\kappa^{*}{ }_{v, l}$ is the absorption coefficient, including induced emission, resulting from the molecular band system with an upper state $i$. The function $F_{v}$ is the incoming radiative intensity integrated over all directions, which may be written as

$$
F_{v}=2 \pi \int_{0}^{\pi} I_{v}(\phi) \sin \phi d \phi \quad\left(\frac{e r g}{c^{2}}\right)
$$


where $I_{v}$ is the directional radiative intensity (erg/ $\left.\mathrm{cm}^{2}-\mathrm{sr}\right)$ and $\phi$ is the angle from the vertical axis directed away from the wall. Note that if a $\cos \phi$ was added to the integrand, the conventional radiative flux equation would be obtained. The evaluation of this term, consistent with the tangent-slab approximation, and written in terms of approximate exponential integrals, is presented in Appendix D of Ref. 32. A significant property of the escape factor is that it depends on the radiation emitted from every point in the shock layer. Therefore if an exact solution is desired, it is necessary to iterate over the entire flowfield. Fortunately, as written in Eq. (9), it is not very dependent on this iteration procedure because of the large value of $N_{i}$ present in the denominator. After evaluating the escape factor exactly through an iteration procedure for the trajectory points considered in this study, it was found that it could be correlated as a function of only temperature as follows:

$$
\begin{array}{ll}
\Lambda_{i} & =-4.6079 \times 10^{-8} T_{v e}^{2}+8.052 \times 10^{-4} T_{v e}-2.6814 \quad 9000 K>T_{v e}>4477 K \\
\Lambda_{i} & =0.0 \quad T_{v e}<4477 K
\end{array}
$$

This correlation is compared with the computed values in Figure 12. Although some error is introduced by using the correlation, it allows for a considerable reduction in computational time and complexity, especially for radiation coupled solutions. A comparison between the radiative flux values computed using the exact values and the correlation is presented below.

The solution to Eq. (3) may be obtained conveniently by applying the quasi-steady state assumption $^{28}$. This assumption assumes that derivative term may be set equal to zero, which transforms the differential equation into an algebraic equation. Both Raynaud et al..$^{5}$ and Magin et al. ${ }^{6}$ investigated the accuracy of this assumption for Titan and concluded that it was sufficiently accurate. Combining Eqs. (3), (4), (8), and the set of excitation rates listed in Table 6, the number densities of the A and B states of the $\mathrm{CN}$ molecule may be solved for, resulting in: 


$$
N_{C N(B)}=N_{C N(X)}\left(\frac{k_{f, 2} N_{M}+k_{f, 4} N_{e}+k_{f, 6} N_{N_{2}(X, v=11)}+k_{f, 7} N_{N_{2}(A)}}{k_{b, 2} N_{M}+k_{b, 4} N_{e}+k_{b, 6} N_{N_{2}(X, v=11)}+k_{b, 7} N_{N_{2}(A)}+\Lambda_{C N(B)} N_{a} / \tau_{C N(B)}}\right)
$$

and

$$
N_{C N(A)}=N_{C N(X)}\left(\frac{k_{f, 1} N_{M}+k_{f, 3} N_{e}+k_{f, 5} N_{N_{2}(X, v=11)}}{k_{b, 1} N_{M}+k_{b, 3} N_{e}+k_{b, 5} N_{N_{2}(X, v=11)}+\Lambda_{C N(A)} N_{a} / \tau_{C N(A)}}\right)
$$

The ground state number density, $\mathrm{CN}(\mathrm{X})$, may be solved from the requirement that the sum of the various state number densities equals the total $\mathrm{CN}$ number density:

$$
N_{C N(X)}=N_{C N}-N_{C N(A)}-N_{C N(B)}
$$

These equations may be set up as a set of three linear equations in three unknowns and solved easily. For the cases considered here, the $\mathrm{A}$ and $\mathrm{X}$ states are so close to their Boltzmann values that Eqs. (13) and (14) do not need to be considered. Therefore, only Eq. (12) is evaluated assuming $N_{C N(X)}$ is equal to its Boltzmann value, along with all of the $\mathrm{N}_{2}$ states.

The influence of the various excitation mechanisms on the $\mathrm{CN}(\mathrm{B})$ number density profile for the $t=189$ case are shown in Figure 13. The "all reactions" case with $\Lambda=1$ (which means that absorption is not included) represents the baseline case. The influence of each reaction is then seen by removing each reaction individually from the calculation and observing the deviation of the predicted values of $N_{C N(B)}$ from the baseline case. This is shown in Figure 13 for reactions 2, 6, and 7, which are defined in Table 6. The most important reaction appears to be reaction 6 , which is resonant-collisional deexcitation reaction. Also shown in this figure is the influence of including the absorption term, which implies using an escape factor less than one. The " $\Lambda=$ exact" case implemented the exact escape factor calculation as described previously. The " $\Lambda=$ fit" case applied Eq. (11) for the escape factor. It is seen that the "exact" and "fit" cases are in 
good agreement. This is also true for the other two trajectory points considered in this study, which therefore validates the use of the curve-fit for these conditions.

The wall radiative heat flux values obtained using the CR model are listed in Table 8 . The results obtained by using the escape factor curve fit or ignoring absorption $(\Lambda=1)$ may be compared with exact escape factor case. It is seen that, again, good agreement is obtained using the curve fit. The reduction in $q_{r}$ due to the use of the CR model, rather than a Boltzmann distribution, is found to be as large as $70 \%$ at $t=185$. The increase in $q_{r}$ due to absorption is found to be the largest at $t=193$, where it provides an increase of about $16 \%$ above the $\Lambda=1$ case. The effect of radiation-flowfield coupling on the CR model was also considered and the resulting radiative heating values presented in the last column of Table 8 . Considering the greatly reduced radiation predicted by the $\mathrm{CR}$ model, it is not surprising that the coupling effect is small. Note that the coupled results, which include the curve-fit model for the escape factor, are not even reduced back to the uncoupled cases with no absorption $(\Lambda=1)$.

It was shown in this section that accounting for a non-Boltzmann distribution reduces the radiative heating by about half. This significant reduction is dependent on the rates used for the various collisional excitation processes, which are not very well known. As shown in Figure 13, reaction 6 is the most important while reactions 2 and 7 are of roughly equal importance. The rate for reaction 2 used in the present model, following past Titan studies, was taken from Zalogin et al. ${ }^{25}$ This study conducted experiments in a $9.6 \% \mathrm{CO}_{2}, 0.3 \% \mathrm{~N}_{2}$ and $90.1 \% \mathrm{Ar}$ freestream at 1 Torr and chose values for the excitation rates so that their computational and experimental results agreed. A concern in using the rate derived from this study is the large argon concentration present, which essentially implies that the collision partner in their experiment for reaction 2 was argon. The non-Boltzmann results obtained at relatively large 
initial pressures indicate that $\mathrm{Ar}$ may be inefficient, relative to $\mathrm{N}_{2}$, at exciting CN. Fairbairn ${ }^{29}$ found a similar result for the excitation of $\mathrm{CN}$ through collisions with argon. Past studies that have considered the excitation of $\mathrm{CN}$ through collisions with $\mathrm{N}_{2}$ have suggested much larger rate constants $^{30,31}$. Unfortunately, these studies have been at low temperature conditions (around 300 K) so that the derived rate constants are not directly applicable to the present problem. It should be mentioned, however, that the rate for reactions $6-8$ were obtained from similar low temperature conditions. Thus, it is worth considering the effect of replacing reaction 2 with one of these low temperature rates. If a value of $1.2 \times 10^{13} \mathrm{~cm}^{3} / \mathrm{mol} / \mathrm{s}$ is used for reaction 2 , as was suggested by Tereshchenko ${ }^{31}$ (this is also close to value suggested by Provencher ${ }^{30}$ ), the resulting $\mathrm{CN}(\mathrm{B})$ populations are very close to a Boltzmann distribution for the cases considered here. This conclusion indicates that caution must be used when considering the large decrease in the radiative heating resulting from the present $\mathrm{CR}$ model.

\section{Conclusions}

An approach to calculating the Huygens probe radiative heating was developed, which included the effects of radiation-flowfield coupling and non-Boltzmann electronic state populations. The ability to treat radiation-flowfield coupling, with reasonable computational requirements, was made possible by using a modified smeared rotational band (SRBC) calculation for the radiations spectrum. This approach was shown to provide frequencyintegrated heat flux values within $5 \%$ of a line-by-line calculation over the range of conditions for Huygens entry. The reduction in radiative heating due to coupling was found to be about $20 \%$ for the three peak-heating trajectory points considered. This reduction was less than that estimated in previous studies using the Tauber-Wakefield correction. The convective heating was shown to be reduced by about $15 \%$. The uncoupled radiative heating, obtained with the efficient 
SRBC method, was shown to agree well with previous NEQAIR results. Details of implementing a collisional-radiative (CR) model for Titan entry were presented. The treatment of the radiation absorption term was formulated in terms of an escape factor. For the range of conditions considered, it was shown that this term could be approximated using a curve-fit as a function of temperature. The use of the $\mathrm{CR}$ model for calculating the population of the $\mathrm{CN}(\mathrm{B})$ state was shown to reduce the radiative heating by roughly a half, with the effect of coupling being small. However, caution is noted in accepting this conclusion because of the uncertainties in the rate data.

\section{References}

${ }^{1}$ Hollis, B.R., Striepe, S.A., Wright, M.J., Bose, D., Sutton, K., and Takashima, N., "Prediction of the Aerothermodynamic Environment of the Huygens Probe," AIAA Paper 2005-4816, June 2005.

${ }^{2}$ Wright, M.J., Olejniczak, J., Walpot, L., Raynaud, E., Magin, T., Caillaut, L., and Hollis, B.R., “A Code Calibration Study for Huygens Entry Aeroheating,” AIAA Paper 2006-382, Jan. 2006.

3 Tauber, M., and Wakefield, R., "Heating Environment and Protection During Jupiter Entry," Journal of Spacecraft and Rockets, Vol. 8, 1971, pp. 630-636.

${ }^{4}$ Bose, D., Wright, M.J., Bogdanoff, D.W., Raiche, G.A., and Allen, G.A., "Modeling and Experimental Validation of CN Radiation Behind a Strong Shock Wave," Journal of Thermophysics and Heat Transfer, Vol. 20, No. 2, 2006, pp. 220-230.

${ }^{5}$ Raynaud, E., Tran, Ph., Soler, J., Baillion, M., "Huygens Aerothermal Environment: Radiative Heating,” ${ }^{\text {rd }}$ International Planetary Probe Workshop, Anavyssos, Greece, 2005. 
${ }^{6}$ Magin, T.E., Caillault, L., Bourdon, A., and Laux, C.O., "Nonequilibrium Radiative Heat Flux Modeling for the Huygens Entry Probe," Journal of Geophysical Research, Vol. 111, 2006, E07S12.

${ }^{7}$ Davis, R.T.,"Numerical Solution of the Hypersonic Viscous Shock Layer Equations," AIAA Journal, Vol. 8, 1970, pp. 843-851.

${ }^{8}$ Moss, J.N. "Reacting Viscous-Shock-Layer Solutions with Multicomponent Diffusion and Mass Injection,” NASA TR R-411, 1974.

${ }^{9}$ Gocken, T., "N ${ }_{2}-\mathrm{CH}_{4}-\mathrm{Ar}$ Chemical Reaction Model for Simulations of Atmospheric Entry to Titan,” AIAA Paper 2004-2469, Jan. 2004.

${ }^{10}$ Park, C., "Assessment of a Two-Temperature Kinetic Model for Dissociating and Weakly Ionizing Nitrogen," Journal of Thermophysics and Heat Transfer, Vol. 2, Jan. 1988, pp. 8-16.

${ }^{11}$ Gnoffo, P.A., Gupta, R.N., Shinn, J. L., "Conservation Equations and Physical Models for Hypersonic Air Flows in Thermal and Chemical Nonequilibrium,” NASA TP 2867, 1989.

${ }^{12}$ Gupta, R.N., and Lee, K.P., "Thermo-Chemical Nonequilibrium Analysis of Viscous ShockLayers," AIAA Paper 95-2083, 1995.

${ }^{13}$ Gupta, R. N., "Viscous Shock-Layer Study of Thermochemical Nonequilibrium," Journal of Thermophysics and Heat Transfer," Vol. 10, No. 2, 1996, pp. 257-266.

${ }^{14}$ Sutton, K., Gnoffo, P. A.,"Multi-Component Diffusion with Application to Computational Aerothermodynamics," AIAA Paper 98-2575, 1998.

${ }^{15}$ Arnold, J. O., Whiting, E. E., "Line by Line Calculation of Spectra from Diatomic Molecules and Assuming a Voigt Line Profile," Journal of Quantitative Spectroscopy and Radiative Transfer, Vol. 9, 1969, pp. 775-798. 
${ }^{16}$ Park, C., "Nonequilibrium Air Radiation (NEQAIR) Program: User's Manual”, NASA TM 86707, 1985.

${ }^{17}$ Whiting, E. E., et al., "NEQAIR96, Nonequilibrium and Equilibrium Radiative Transport and Spectra Program: User's Manual,” NASA RP 1389, Dec. 1996, pp. 91-98.

${ }^{18}$ Patch, R.W., Shackleford, W.L., Penner, S.S, “Approximate Spectral Absorption Coefficient Calculations for Electronic Band Systems Belonging to Diatomic Molecules," Journal of Quantitative Spectroscopy and Radiative Transfer, Vol. 2, July-Sept. 1962, pp. 263-271.

${ }^{19}$ Hartung, L.C., "Development of a Nonequilibrium Radiative Heating Prediction Method for Coupled Flowfield Solutions", Journal of Thermophysics and Heat Transfer, Vol. 6, pp. 618$625,1992$.

${ }^{20}$ Chambers, L. H., "Predicting Radiative Heat Transfer in Nonequilibrium Flow Fields: Theory and User's Manual for the LORAN Code," NASA TM 4564, Sept. 1994.

${ }^{21}$ Laux, C.O., "Optical Diagnostics and Radiative Emission of Air Plasmas," High Temperature Gasdynamics Laboratory Report No. T-288, Stanford University, August 1993.

${ }^{22}$ Olejniczak, J., Wright, M.J., Prabhu, D., Takashima, N., Hollis, B.R., Zoby, E.V., and Sutton, K., "An Analysis of the Radiative Heating Environment for Aerocapture at Titan," AIAA Paper 2003-4953, 2003.

${ }^{23}$ Mazoue, F., Marraffa, L., "Flow-field/Radiation Coupling Analysis for Huygens Probe Entry into Titan Atmosphere,” AIAA Paper 2005-5392, June 2005.

${ }^{24}$ Wright, M.J., Bose, D., Olejniczak, J., “The Impact of Flowfield-Radiation Coupling on Aeroheating for Titan Aerocapture," Journal of Thermophysics and Heat Transfer, Vol. 19, Jan.March 2005, pp. 17-27. 
${ }^{25}$ Zalogin, G.N., Koslov, P.V., Kuznetsova, L.A., Losev, S.A., Makarov, V.N., Romanenko, Y.P., and Surzhikov, S.T., "Radiation Excited by Shock Waves in a $\mathrm{CO}_{2}-\mathrm{N}_{2}-\mathrm{Ar}$ Mixture: Experiment and Theory," Technical Physics, Vol. 46, No. 6, 2001.

26 Kudryavtsev, N.N., Kuznetsova, L.A., Surzhikov, S.T., "Kinetics and Nonequilibrium Radiation of $\mathrm{CO}_{2}-\mathrm{N}_{2}$ Shock Waves,” AIAA Paper 2001-2728, June 2001.

${ }^{27}$ Nelson, H.F., Park, C., Whiting, E.E., “Titan Atmospheric Composition by Hypervelocity Shock-Layer Analysis," Journal of Thermophysics and Heat Transfer, Vol. 5, 1991, pp. 157165.

${ }^{28}$ Park, C., Nonequilibrium Hypersonic Aerothermodynamics, John Wiley and Sons, New York, 1990, pp. 89-97.

${ }^{29}$ Fairbairn, A.R., "Shock-Tube Study of the Dissociation Rate of CN," The Journal of Chemical Physics, Vol. 51, August 1969, pp. 972-975.

${ }^{30}$ Provencher, G.M., McKenney, D.J., 'CN Emission in Active Nitrogen. II. The Role of Energy Transfer and Atom Transfer Reactions in $\mathrm{CN}\left(\mathrm{X}^{2} \Sigma^{+}\right)$Excitation," Canadian Journal of Chemistry, Vol. 50, 1972, pp. 2527-2536.

31 Tereshchenko, E.N., and Dodonova, N.A., "Collisional Deactivation Processes for Electronically Excited CN(B $\left.{ }^{2} \Sigma^{+}\right)$Radicals," Optics and Spectroscopy, Vol. 39, Oct. 1975, pp. $435-436$.

32 Johnston, C. O., Hollis, B. R., Sutton, K., "Radiative Heating Methodology for the Huygens Probe,” AIAA Paper 2006-3426, June 2006. 
$\underline{\text { Tables }}$

Table 1. Huygens entry conditions considered in this study

\begin{tabular}{cccc}
\hline$t(\mathrm{~s})$ & $U_{\text {inf }}(\mathrm{m} / \mathrm{s})$ & $\rho_{\text {inf }}\left(\mathrm{kg} / \mathrm{m}^{3}\right)$ & $T_{\text {inf }}(\mathrm{K})$ \\
\hline 185 & 5489 & $1.83 \times 10^{-4}$ & 177.0 \\
189 & 5126 & $2.96 \times 10^{-4}$ & 176.6 \\
193 & 4705 & $3.79 \times 10^{-4}$ & 175.8 \\
\hline
\end{tabular}

Table 2 . Intensity resulting from a $1-\mathrm{cm}$ constant property slab of $\mathrm{CN}$ in equilibrium at $7000 \mathrm{~K}\left(\mathrm{~W} / \mathrm{cm}^{2}-\mathrm{sr}\right)$

\begin{tabular}{ccccc}
\hline$p(\mathrm{~atm})$ & $N_{C N}\left(\mathrm{~cm}^{-3}\right)$ & NEQAIR96 & Present - LBL & Present - SRB \\
\hline 0.001 & $1.05 \times 10^{15}$ & 1.67 & 1.60 & 1.63 \\
0.01 & $1.05 \times 10^{16}$ & 11.7 & 11.6 & 14.3 \\
0.1 & $1.05 \times 10^{17}$ & 41.0 & 39.6 & 60.3 \\
\hline
\end{tabular}

Table 3. Breakdown of the radiative heat flux towards the wall $\left(q_{r}^{-}\right)$at $z=0$ for the uncoupled case $\left(\mathrm{W} / \mathrm{cm}^{2}\right)$, values not is parenthesis are the SRBC predictions and values in parenthesis are the LBL predictions.

\begin{tabular}{|c|c|c|c|c|c|c|c|}
\hline$t(\mathrm{sec})$ & $\begin{array}{c}\mathrm{CN} \text { violet } \\
\Delta V=0\end{array}$ & $\begin{array}{c}\mathrm{CN} \text { violet } \\
\Delta V=1\end{array}$ & $\begin{array}{c}\mathrm{CN} \text { violet } \\
\Delta V=-1\end{array}$ & $\begin{array}{l}\mathrm{CN} \\
\text { red }\end{array}$ & Total & $\begin{array}{l}\text { NEQAIR } \\
\text { (Ref. 1) }\end{array}$ & $\begin{array}{c}\text { RAD/EQUIL } \\
\text { (Ref. 1) }\end{array}$ \\
\hline 185 & $46.7(47.7)$ & $11.6(12.6)$ & $9.0(9.5)$ & 4.4 & $71.7(74.2)$ & 72.3 & 118.4 \\
\hline 189 & $50.5(50.7)$ & $13.5(14.8)$ & $11.3(11.8)$ & 7.5 & $82.8(84.8)$ & 81.5 & 143.1 \\
\hline 193 & $39.8(39.3)$ & $11.3(12.7)$ & $10.3(10.7)$ & 10.5 & $71.9(73.2)$ & 72.1 & 147.2 \\
\hline
\end{tabular}

Table 4. Comparison of the convective heat flux for the uncoupled and coupled cases $\left(\mathrm{W} / \mathrm{cm}^{2}\right)$

\begin{tabular}{ccc|c}
\hline$t(\mathrm{sec})$ & $\begin{array}{c}q_{c} \\
\text { uncoupled }\end{array}$ & $\begin{array}{c}q_{c} \\
\text { coupled }\end{array}$ & $\begin{array}{c}\% \\
\text { difference }\end{array}$ \\
\hline 185 & 43.9 & 37.6 & 14.4 \\
189 & 44.5 & 38.7 & 13.0 \\
193 & 37.9 & 33.7 & 11.1 \\
\hline
\end{tabular}


Table 5. Comparison of the radiative heat flux towards the wall at $z=0$ for the uncoupled and coupled cases $\left(\mathrm{W} / \mathrm{cm}^{2}\right)$

\begin{tabular}{ccc|c|c}
\hline$t(\mathrm{sec})$ & $\begin{array}{c}q_{r}^{-} \\
\text {uncoupled }\end{array}$ & $\begin{array}{c}q_{r}^{-} \\
\text {coupled }\end{array}$ & $\%$ difference & $\begin{array}{c}q_{r}^{-} \\
\text {coupled - TW }\end{array}$ \\
\hline 185 & $71.7(74.2)$ & $59.0(60.8)$ & $17.7(18.1)$ & $51.8(53.2)$ \\
189 & $82.8(84.8)$ & $66.5(68.3)$ & $19.7(19.5)$ & $61.3(62.5)$ \\
193 & $71.9(73.2)$ & $54.0(55.0)$ & $25.0(25.0)$ & $54.5(55.3)$ \\
\hline
\end{tabular}

Table 6. Excitation reactions considered in the present study

\begin{tabular}{lccccccc}
\hline & $A$ & $n$ & $C$ & $T_{f}$ & $T_{b}$ & Ref. \\
\hline & Neutral Particle Collisional Deexcitation: & & & & & & \\
1 & $\mathrm{CN}(\mathrm{X})+M \leftrightarrow \mathrm{CN}(\mathrm{A})+M$ & $1.5 \mathrm{e}+11$ & 0.5 & 13300 & $T_{a}$ & $T_{t r}$ & Zagolin \\
2 & $\mathrm{CN}(\mathrm{X})+M \leftrightarrow \mathrm{CN}(\mathrm{B})+M$ & $1.8 \mathrm{e}+11$ & 0.5 & 37000 & $T_{a}$ & $T_{t r}$ & Zagolin \\
& Electron Impact Deexcitation: & & & & & & \\
3 & $\mathrm{CN}(\mathrm{X})+\mathrm{e}^{-} \leftrightarrow \mathrm{CN}(\mathrm{A})+\mathrm{e}^{-}$ & $6.0 \mathrm{e}+14$ & 0.5 & 13300 & $T_{v e}$ & $T_{v e}$ & Zagolin \\
4 & $\mathrm{CN}(\mathrm{X})+\mathrm{e}^{-} \leftrightarrow \mathrm{CN}(\mathrm{B})+\mathrm{e}^{-}$ & $6.3 \mathrm{e}+14$ & 0.5 & 37000 & $T_{v e}$ & $T_{v e}$ & Zagolin \\
& Resonant Collisional Deexcitation: & & & & & & \\
5 & $\mathrm{CN}(\mathrm{X})+\mathrm{N}_{2}(\mathrm{X}, V=4) \leftrightarrow \mathrm{CN}(\mathrm{A})+\mathrm{N}_{2}(\mathrm{X}, V=0)$ & $6.0 \mathrm{e}+13$ & 0 & 0 & - & $T_{t r}$ & Chernyi \\
6 & $\mathrm{CN}(\mathrm{X})+\mathrm{N}_{2}(\mathrm{X}, V=11) \leftrightarrow \mathrm{CN}(\mathrm{B})+\mathrm{N}_{2}(\mathrm{X}, V=0)$ & $6.0 \mathrm{e}+13$ & 0 & 0 & - & $T_{t r}$ & Chernyi \\
& Quenching Reaction: & & & & & & \\
7 & $\mathrm{~N}_{2}(\mathrm{~A})+\mathrm{CN}(\mathrm{X}) \leftrightarrow \mathrm{N}_{2}(\mathrm{X})+\mathrm{CN}(\mathrm{B})$ & $4.2 \mathrm{e}+12$ & 0.5 & 0 & $T_{a}$ & $T_{t r}$ & Pintassilgo \\
\hline
\end{tabular}

Table 7. Radiative lifetimes for the $\mathrm{CN}$ radiative transitions.

\begin{tabular}{lcc}
\hline & $\tau(s)$ & Ref. \\
\hline $\mathrm{CN}(\mathrm{A}) \rightarrow \mathrm{CN}(\mathrm{X})+h v$ & $1.54 \mathrm{e}-5$ & Zagolin \\
$\mathrm{CN}(\mathrm{B}) \rightarrow \mathrm{CN}(\mathrm{X})+h v$ & $6.55 \mathrm{e}-8$ & Zagolin \\
\hline
\end{tabular}

Table 8. Comparison of the radiative heat flux towards the wall at $z=0$ for the various

\begin{tabular}{cccc|c}
\multicolumn{5}{c|}{ escape factor models $\left(\mathrm{W} / \mathrm{cm}^{2}\right)$} \\
\hline$t(\mathrm{sec})$ & $\begin{array}{c}q_{r} \\
\text { uncoupled } \\
(\Lambda=\text { exact })\end{array}$ & $\begin{array}{c}q_{r} \\
\text { uncoupled } \\
(\Lambda=\mathrm{fit})\end{array}$ & $\begin{array}{c}q_{r} \\
\text { uncoupled } \\
(\Lambda=1.0)\end{array}$ & $\begin{array}{c}q_{r} \\
\text { coupled } \\
(\Lambda=\mathrm{fit})\end{array}$ \\
\hline 185 & 21.0 & $21.4(22.0)$ & 18.2 & 20.8 \\
189 & 35.3 & $34.6(35.4)$ & 29.0 & 32.8 \\
193 & 40.3 & $39.3(39.9)$ & 31.1 & 34.9 \\
\hline
\end{tabular}




\section{$\underline{\text { Figures }}$}

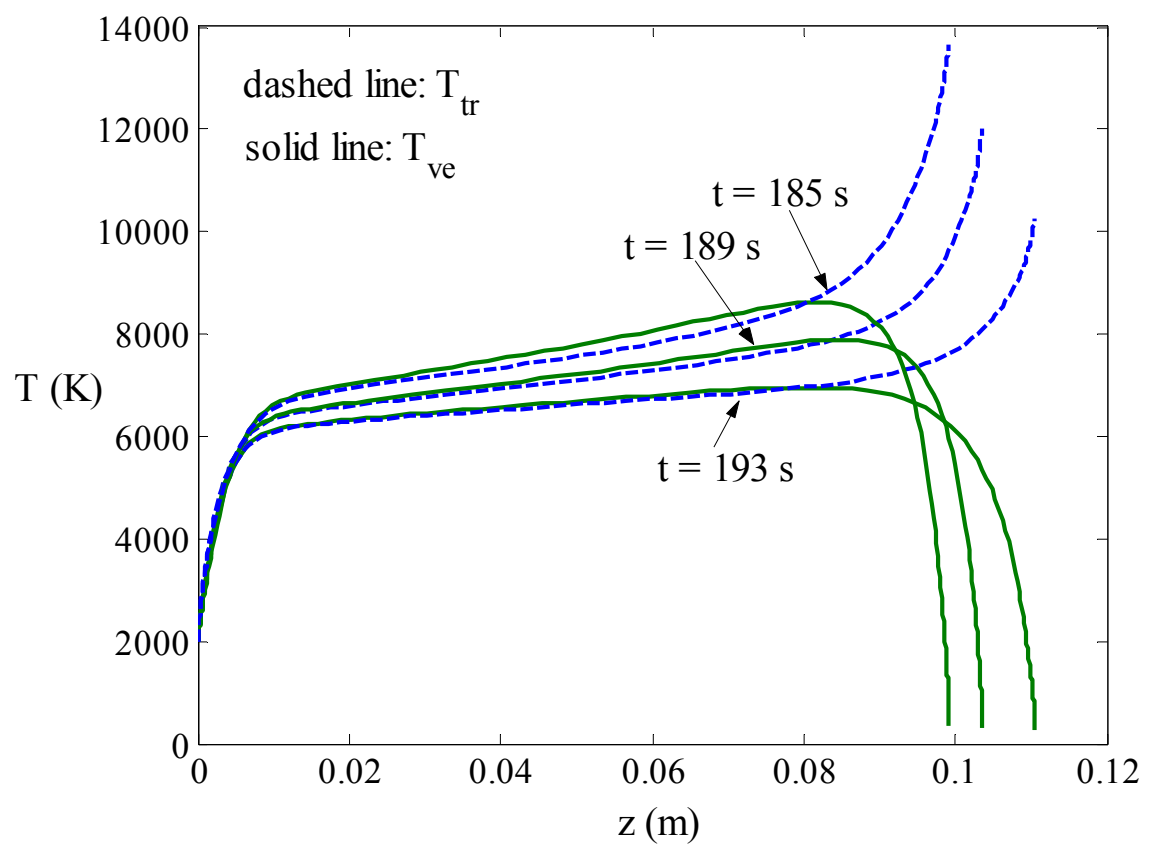

Figure 1. Stagnation line temperature profiles from the viscous shock layer solution at three peak heating trajectory points.

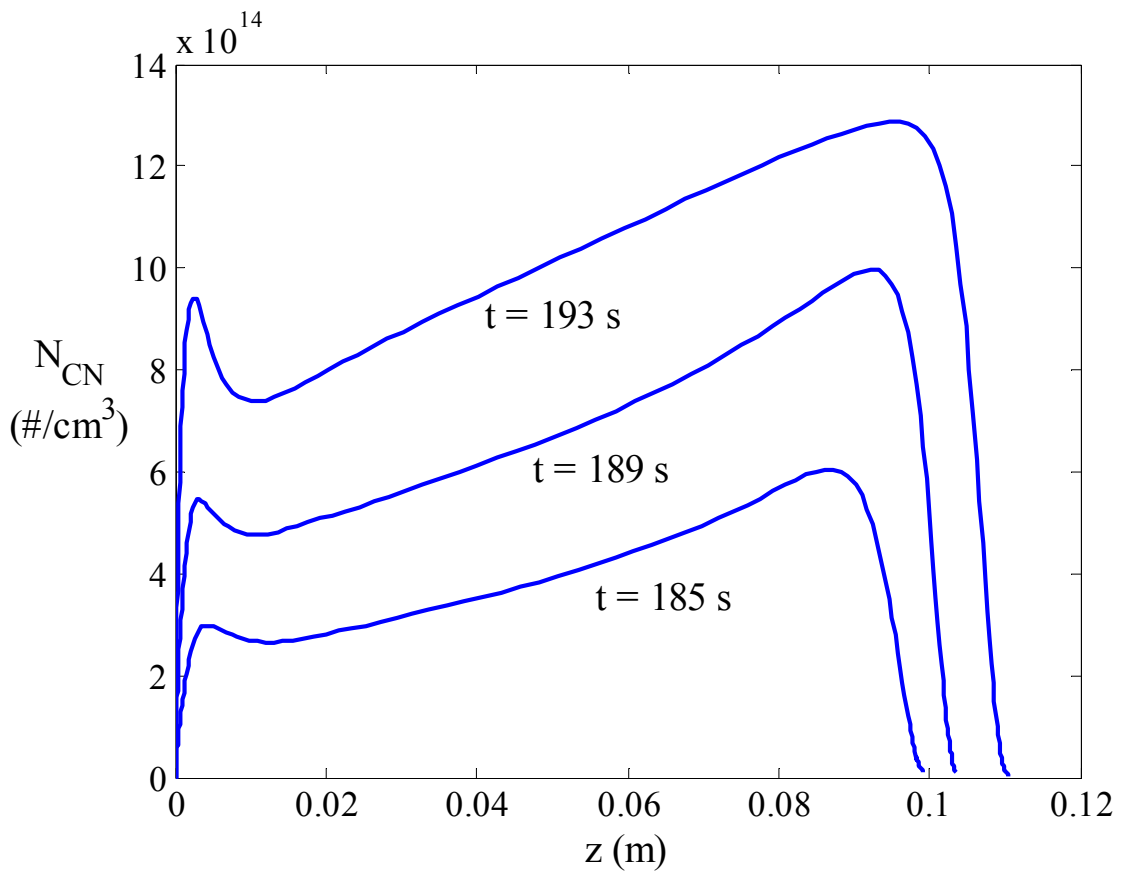

Figure 2. Stagnation line $\mathrm{CN}$ number density profiles at three peak heating trajectory points. 


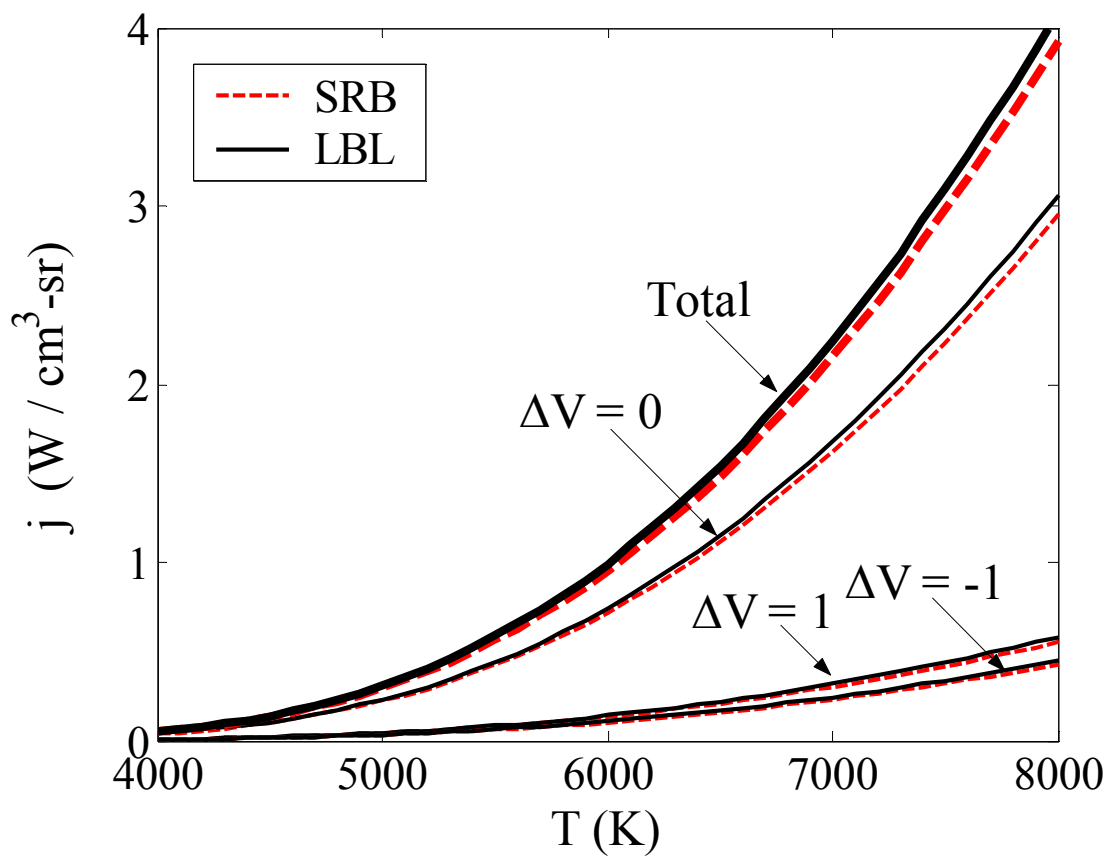

Figure 3. Comparison of the LBL and SRB predictions for the $C N$ violet integrated emission coefficient for $N_{C N}=1 \times 10^{15} \mathrm{~cm}^{-3}$
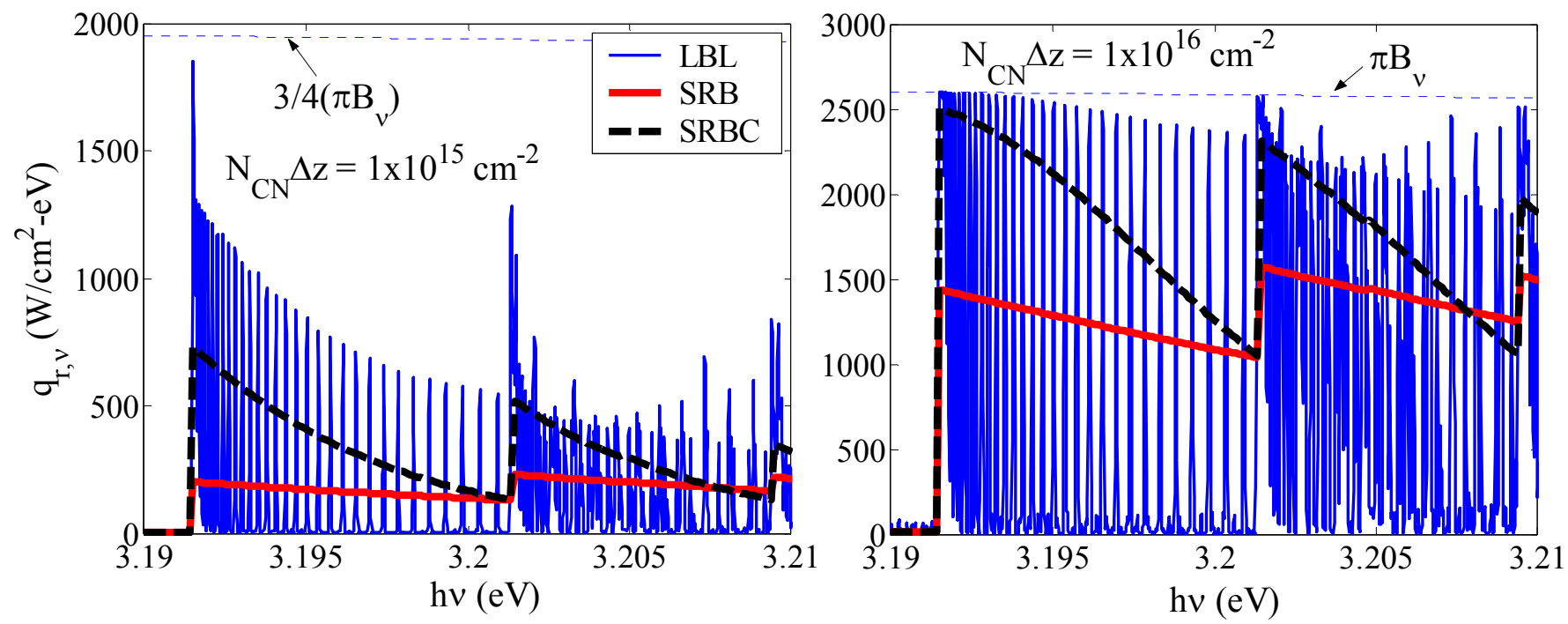

Figure 4. Comparison between the various spectrum models for the radiative flux spectrum from a constant property slab of $\mathrm{CN}$ at $7000 \mathrm{~K}$. The spectral range shown illustrates the (0-0) and (1-1) band heads. 


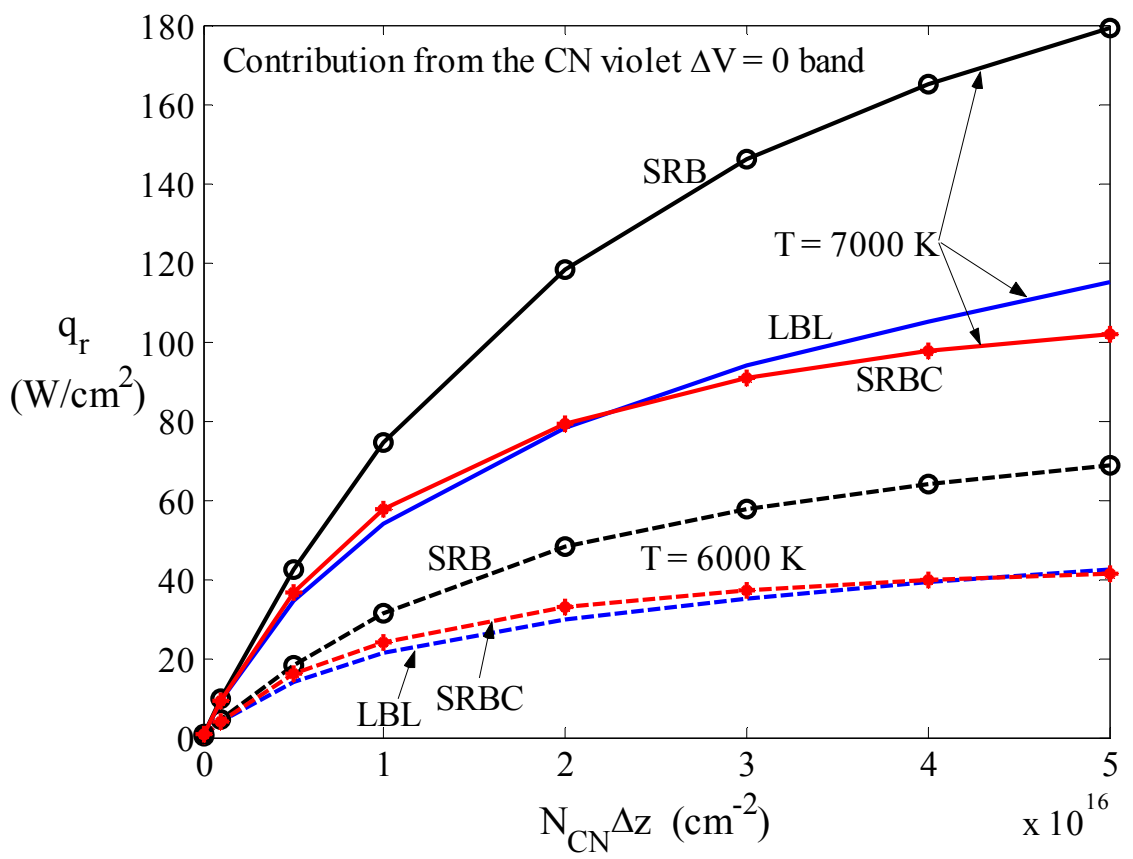

Figure 5. Comparison of the radiative flux from a constant property slab for different spectral models.

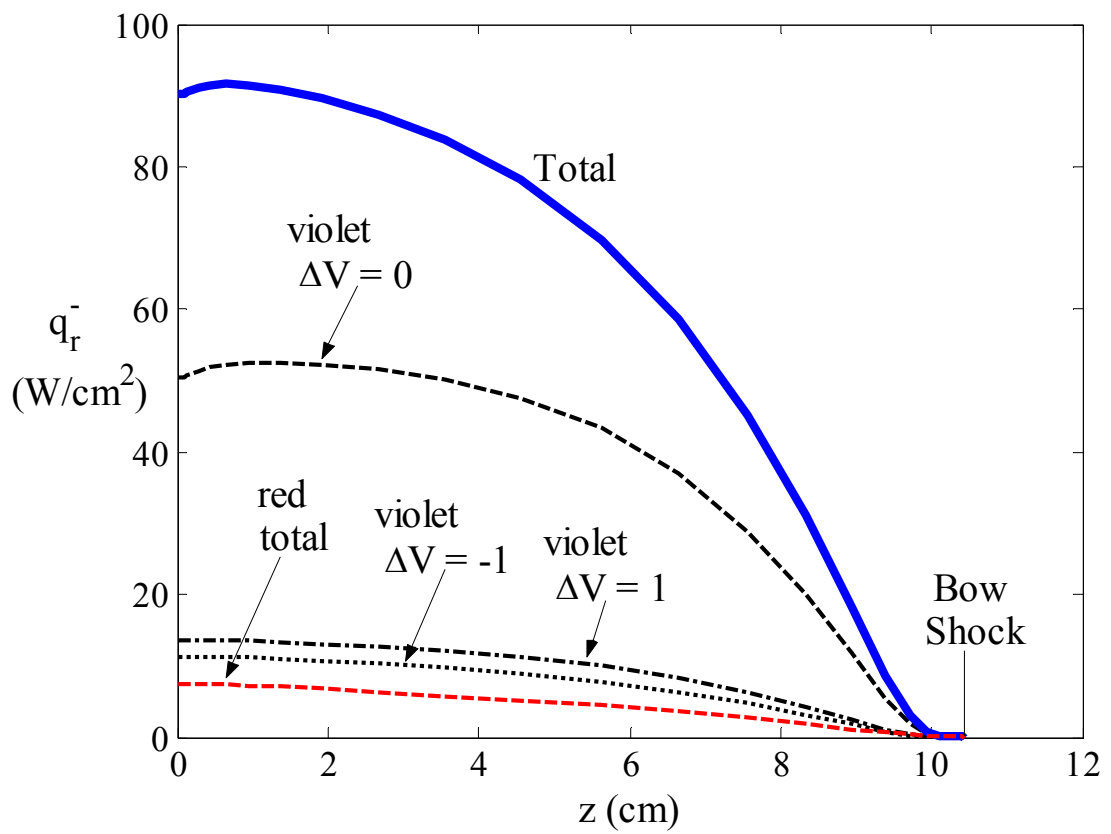

Figure 6 . The contributions from the various radiative mechanisms to the wall directed radiative flux profile for the $t=189$ uncoupled case 


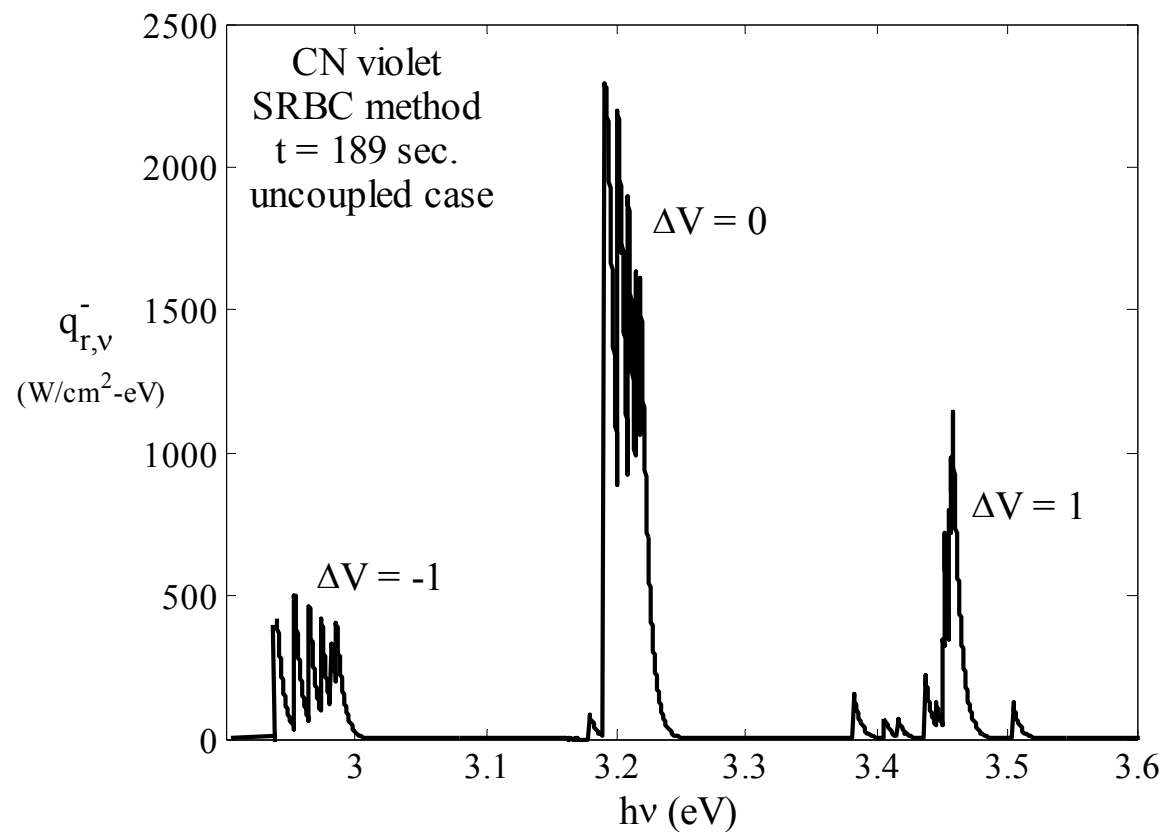

Figure 7. The $\mathrm{CN}$ violet spectrum at the wall as predicted by the SRBC method for the $t=$ 189 coupled case.

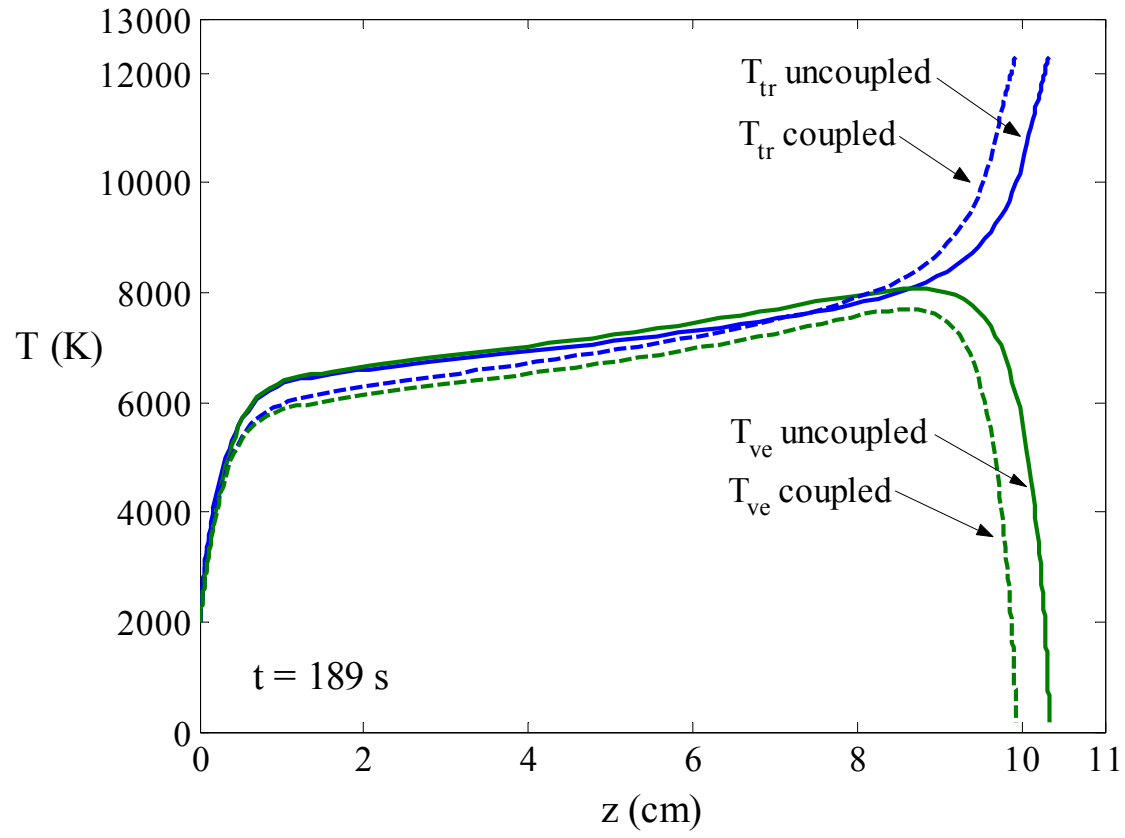

Figure 8. Comparison between the coupled and uncoupled temperature profiles for the $t=189$ case. 


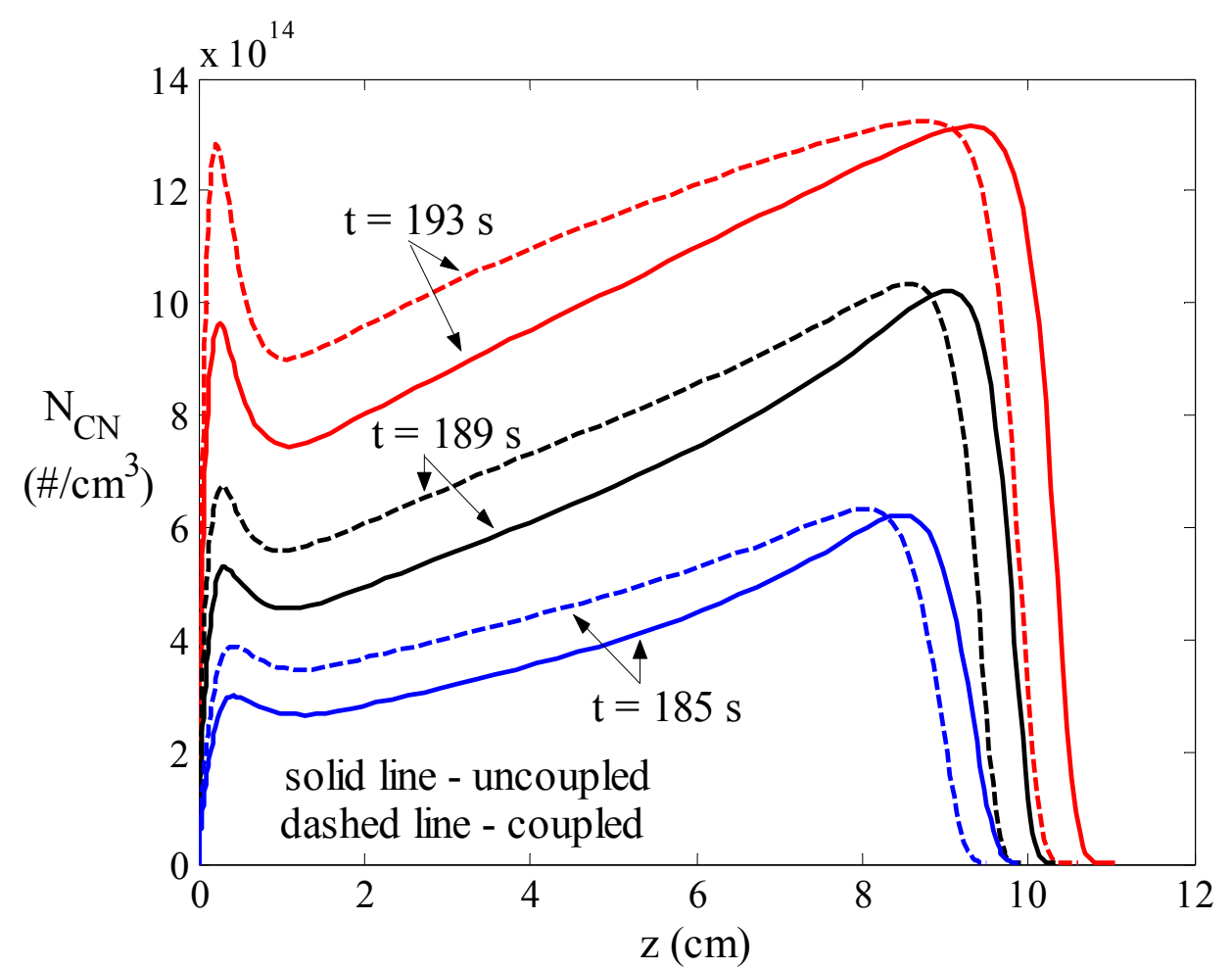

Figure 9. Comparison between the coupled and uncoupled $\mathrm{CN}$ number density profiles.

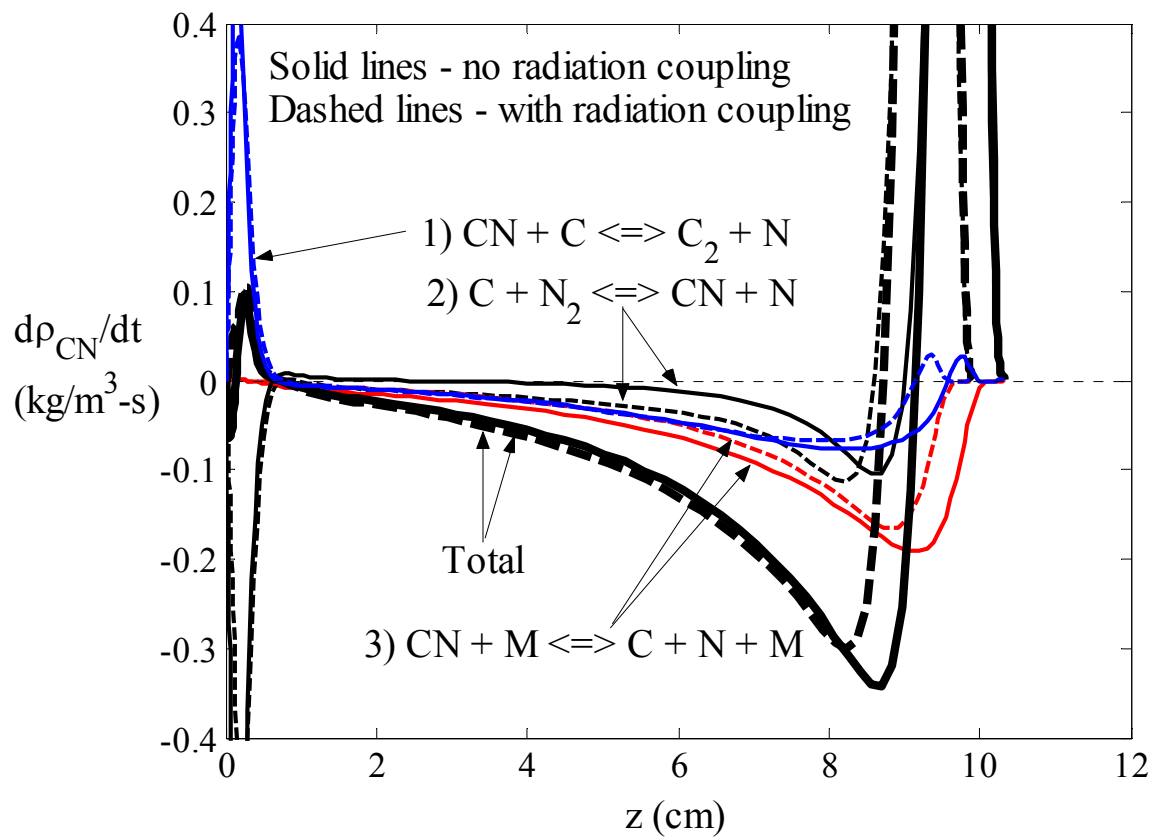

Figure 10. Comparison of the mass rate of formation of $\mathrm{CN}$ molecules by various reactions for the uncoupled and coupled cases at $t=189 \mathrm{~s}$. 


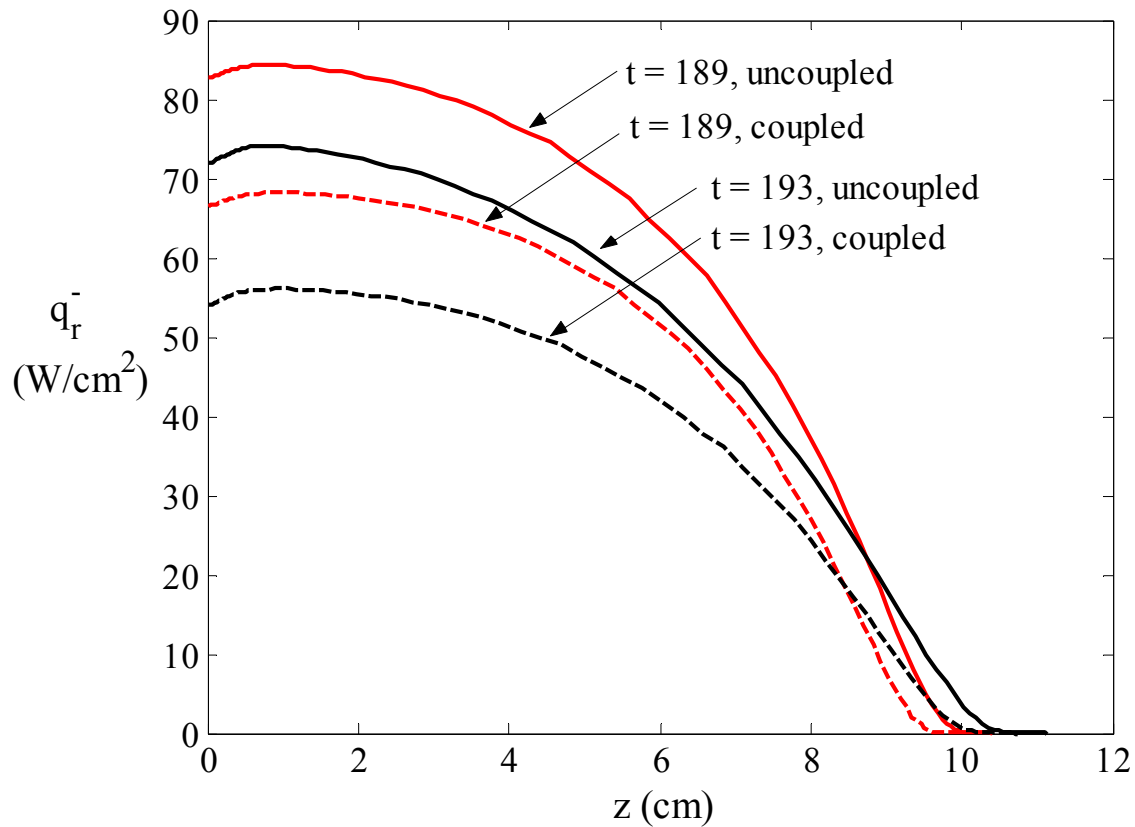

Figure 11. Comparison between the coupled and uncoupled wall-directed radiative flux profiles.

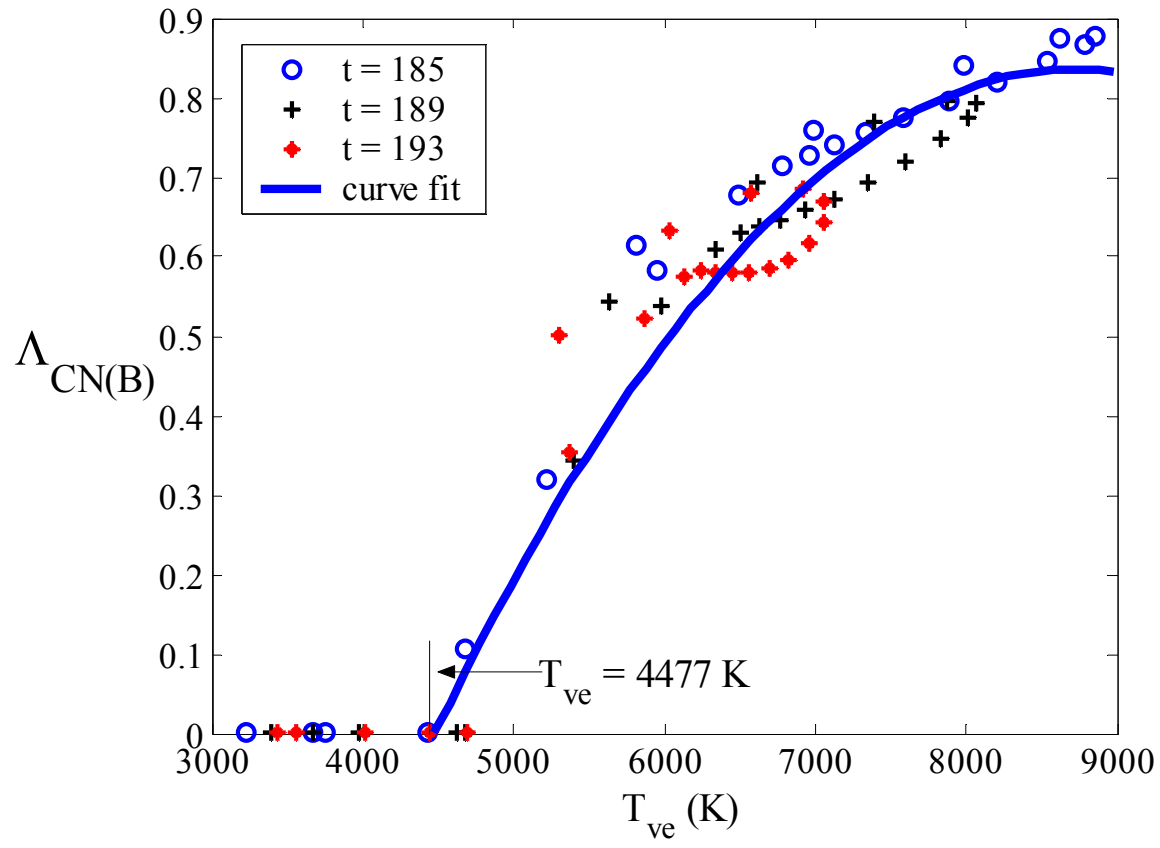

Figure 12. Values of the escape factor calculated for the three trajectory points compared with an approximate curve fit. 


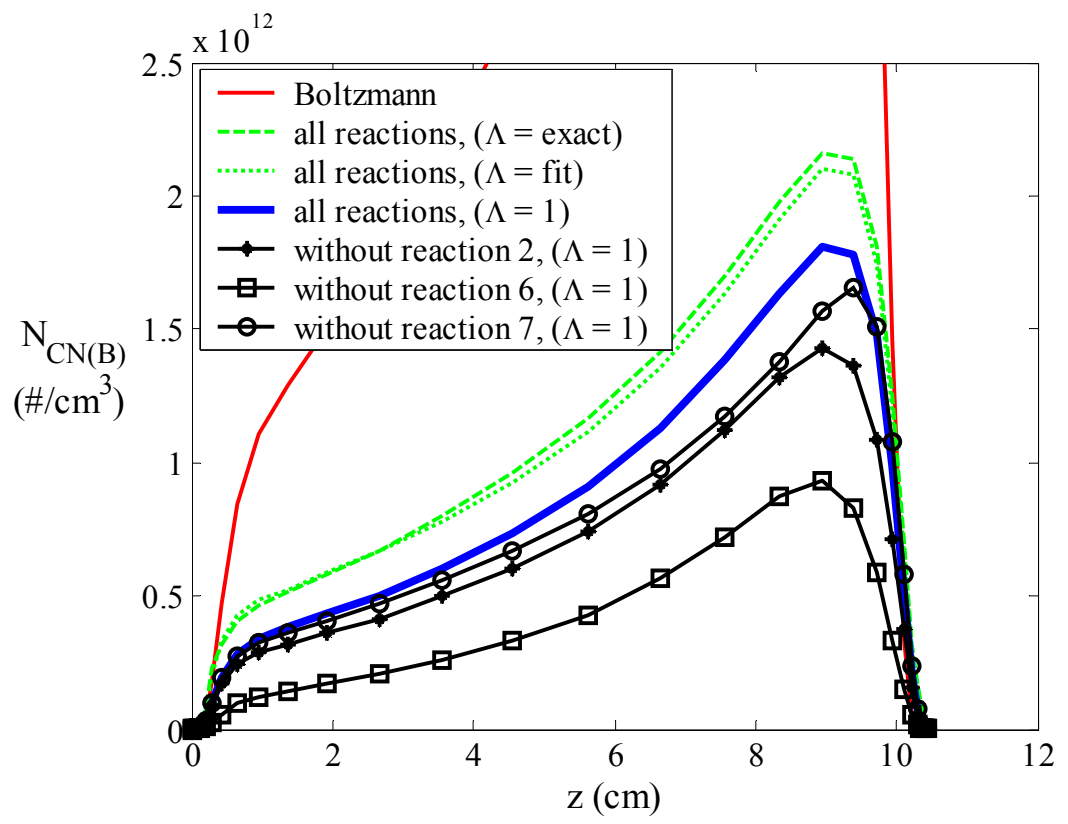

Figure 13. The influence of various excitation mechanisms on the $\mathrm{CN}(\mathrm{B})$ number density for the $\mathrm{t}=189$ case. 